\title{
Sprouty-2 regulates oncogenic K-ras in lung development and tumorigenesis
}

\author{
Alice T. Shaw, ${ }^{1,2,3}$ Alexander Meissner, ${ }^{2,4}$ James A. Dowdle, 1,2 Denise Crowley,, ${ }^{1,5}$ \\ Margaret Magendantz, ${ }^{1,2}$ Chensi Ouyang, ${ }^{1,2}$ Tiziana Parisi, ${ }^{1,2}$ Jayaraj Rajagopal, ${ }^{6}$ Leah J. Blank, ${ }^{6}$ \\ Roderick T. Bronson, ${ }^{7}$ James R. Stone, ${ }^{8}$ David A. Tuveson, ${ }^{9}$ Rudolf Jaenisch, ${ }^{2,4}$ and \\ Tyler Jacks $1,2,5,10$ \\ ${ }^{1}$ Center for Cancer Research, Massachusetts Institute of Technology, Cambridge, Massachusetts 02139, USA; ${ }^{2}$ Department \\ of Biology, Massachusetts Institute of Technology, Cambridge, Massachusetts 02139, USA; ${ }^{3}$ Massachusetts General Hospital \\ Cancer Center and Harvard Medical School, Boston, Massachusetts 02114, USA; ${ }^{4}$ Whitehead Institute for Biomedical \\ Research, Cambridge, Massachusetts 02142, USA; ${ }^{5}$ Howard Hughes Medical Institute at Massachusetts Institute of \\ Technology, Cambridge, Massachusetts 02139, USA; ${ }^{6}$ Department of Molecular and Cellular Biology, Harvard University, \\ Cambridge, Massachusetts 02138, USA; ${ }^{7}$ Department of Pathology, Tufts University School of Medicine and Veterinary \\ Medicine, Boston, Massachusetts 02111, USA; ${ }^{8}$ Department of Pathology, Massachusetts General Hospital and Harvard \\ Medical School, Boston, Massachusetts 02114, USA; ${ }^{9}$ Cambridge Research Institute, Cambridge CB2 ORE, United Kingdom
}

Somatic activation of Ras occurs frequently in human cancers, including one-third of lung cancers. Activating Ras mutations also occur in the germline, leading to complex developmental syndromes. The precise mechanism by which Ras activation results in human disease is uncertain. Here we describe the phenotype of a mouse engineered to harbor a germline oncogenic K-ras ${ }^{G 12 D}$ mutation. This mouse exhibits early embryonic lethality due to a placental trophoblast defect. Reconstitution with a wild-type placenta rescues the early lethality, but mutant embryos still succumb to cardiovascular and hematopoietic defects. In addition, mutant embryos demonstrate a profound defect in lung branching morphogenesis associated with striking up-regulation of the Ras/mitogen-activated protein kinase (MAPK) antagonist Sprouty-2 and abnormal localization of MAPK activity within the lung epithelium. This defect can be significantly suppressed by lentiviral short hairpin RNA (shRNA)-mediated knockdown of Sprouty-2 in vivo. Furthermore, in the context of K-ras ${ }^{G 12 D}$-mediated lung tumorigenesis, Sprouty-2 is also up-regulated and functions as a tumor suppressor to limit tumor number and overall tumor burden. These findings indicate that in the lung, Sprouty-2 plays a critical role in the regulation of oncogenic K-ras, and implicate counter-regulatory mechanisms in the pathogenesis of Ras-based disease.

[Keywords: K-ras; mouse models; lung development; lung cancer; Sprouty]

Supplemental material is available at http://www.genesdev.org.

Received December 27, 2006; revised version accepted February 5, 2007.

Ras proteins play a critical role in the regulation of normal cellular proliferation as well as in the development of neoplasia. Activating mutations in the three major Ras family members ( $H$-ras, $N$-ras, and $\mathrm{K}$-ras) are found in $\sim 30 \%$ of all human tumors (Bos 1989). Certain tumor types have a particularly high incidence of activating Ras mutations, including pancreatic, colon, and lung carcinomas. These mutations are predominantly missense mutations involving codons 12,13, and 61, and compromise the GTPase activity of Ras, resulting in the accumulation of active, GTP-bound Ras.

Upon activation, Ras proteins engage multiple down-

${ }^{10}$ Corresponding author.

E-MAIL tjacks@mit.edu; FAX (617) 253-9863.

Article is online at http://www.genesdev.org/cgi/doi/10.1101/gad.1526207. stream effectors, leading to a wide range of biological responses, from proliferation to differentiation to apoptosis. Through the aberrant regulation of these effectors, oncogenic Ras proteins mediate several key aspects of malignant transformation, including deregulated cell growth and evasion of apoptosis (Downward 2002). However, the precise roles of individual Ras effectors in transformation and tumorigenesis are poorly understood (Malumbres and Barbacid 2003). For example, mouse embryonic fibroblasts (MEFs) and epithelial hyperplasias expressing physiologic levels of oncogenic K-ras are hyperproliferative, yet show attenuation rather than augmentation of Raf/Mek/Erk or mitogen-activated protein kinase (MAPK) signaling (Guerra et al. 2003; Tuveson et al. 2004). These results highlight the complexity of the Ras signaling network, with differential activation of ef- 
fector pathways depending on cellular context, as well as negative regulation of pathway activity through crosstalk and feedback inhibition.

Whereas the oncogenic properties of Ras proteins have been the subject of intense investigation, relatively few studies have addressed the role of Ras in normal mammalian development. These studies have primarily used gene targeting strategies to disrupt Ras oncogenes, singly and in combination. Although $\mathrm{H}$-ras, $\mathrm{N}$-ras, and $\mathrm{K}$-ras are all widely expressed during embryogenesis, only $\mathrm{K}$ ras is essential for normal mouse development (Johnson et al. 1997). Embryos homozygous for a null mutation of

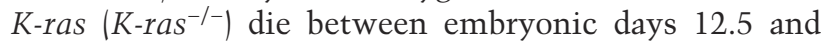
14.5 (E12.5 and E14.5) of anemia and fetal liver defects. These defects may be related to abnormalities in cytokine-induced formation of E-cadherin-based adherens junctions (Matsui et al. 2002), or may be secondary to extraembryonic dysfunction (A. Shaw, unpubl.). In contrast, targeted disruption of $\mathrm{H}$-ras or $\mathrm{N}$-ras, individually and in combination, results in no discernible phenotype (Esteban et al. 2001). While useful in establishing a unique and essential role for K-ras in development (Johnson et al. 1997), this loss-of-function approach has provided limited insight into mechanisms of Ras-mediated transformation.

To date, gain-of-function mutants have not been extensively characterized in the context of early mouse development. Analysis of such mutants would be relevant to the pathogenesis of neoplastic as well as developmental disorders. In particular, several overlapping human genetic syndromes, including Costello, Noonan, and cardio-facio-cutaneous (CFC) syndromes, have recently been shown to be caused by germline activating mutations in Ras and other genes associated with Ras/ MAPK signaling (Aoki et al. 2005; Niihori et al. 2006; Rodriguez-Viciana et al. 2006; Schubbert et al. 2006). While the H-ras mutations identified in Costello syndrome are also found somatically in various tumors (Aoki et al. 2005), the K-ras mutations discovered in Noonan and CFC syndrome are novel and confer distinct gain-of-function properties (Schubbert et al. 2006). These observations suggest that oncogenic K-ras mutations such as $\mathrm{K}$-ras ${ }^{G 12 D}$ are not tolerated during human development. Indeed, we previously demonstrated that expression of $\mathrm{K}$-ras ${ }^{G 12 D}$ in the mouse germline is embryonic lethal, with mutants dying by midgestation (Tuveson et al. 2004).

Here we report the first detailed description of the consequences of oncogenic K-ras activation in the developing mouse. In addition to placental, cardiovascular, and hematopoietic defects, mutant embryos also exhibit a profound defect in lung branching morphogenesis. Using both in vivo RNA interference (RNAi) and traditional genetic approaches, we show that the Ras/MAPK antagonist Sprouty-2 (Spry-2) is a critical mediator of the lung branching phenotype. We also show that Spry-2 functions as a tumor suppressor in $\mathrm{K}$-ras $^{G 12 D}$-mediated lung tumorigenesis. These results highlight the potential involvement of negative regulators of Ras signaling in developmental as well as neoplastic disorders.

\section{Results}

The early embryonic lethality associated with germline oncogenic K-ras is due to extraembryonic dysfunction

We activated expression of oncogenic K-ras in the mouse germline by crossing mice carrying a Protamine-Cre (Prm-Cre) transgene (O'Gorman et al. 1997) with conditional LSL-K-ras ${ }^{G 12 D}$ mice (Jackson et al. 2001). Germline mutants die between E9.5 and E11.5 with developmental arrest and widespread apoptosis (Tuveson et al. 2004). To determine whether extraembryonic dysfunction contributes to this early lethality, we first analyzed the yolk sacs and placentas of E9.5 embryos. On gross examination, mutant yolk sacs appeared pale and roughened, with poorly developed vasculature (Fig. 1A). Whole-mount staining of mutant yolk sacs with antiPECAM antibodies confirmed a primitive, honeycomblike network lacking branching vitelline vessels (Fig. 1B). Although mutant placentas appeared grossly normal, hematoxylin and eosin (H\&E) staining revealed a marked defect in the inner labyrinth layer, with the complete absence of underlying fetal blood vessels (Fig. 1C). This defect in labyrinth vascularization was confirmed by crossing a Tie2-LacZ transgene, which expresses $L a c Z$ specifically in vascular endothelial cells (Schlaeger et al. 1997), into the K-ras ${ }^{G 12 D}$ background (Fig. 1D). Outside the labyrinth, the spongiotrophoblast and trophoblast giant cell layers appeared to be structurally intact, based on $H \& E$ staining and expression patterns of cell-typespecific markers, including P11 (Faria et al. 1991) and Tpbp (Lescisin et al. 1988; data not shown).

The extraembryonic phenotype of K-ras ${ }^{G 12 D}$ mutants suggested a primary defect in vascular endothelial cells. However, LSL-K-ras ${ }^{G 12 D}$;Tie2-Cre compound mutants in which K-ras activation is restricted to the endothelial cell compartment showed normal vascularization of both the yolk sac and the placental labyrinth (Supplementary Fig. 1). To investigate the role of placental trophoblast cells, we crossed LSL-K-ras ${ }^{G 12 D}$ mice with Mox2-Cre transgenic mice (Tallquist and Soriano 2000). LSL-K-ras ${ }^{G 12 D}$;Mox2-Cre compound mutants express Cre under the control of the endogenous Mox 2 promoter, leading to recombination throughout the embryo proper (Wu et al. 2003), but not in the trophoblast or extraembryonic endoderm (Fig. 2A; Supplementary Fig. 2). Thus, this approach enables generation of germline K-ras ${ }^{G 12 D}$ mutants supported by functionally normal placentas.

$L S L-K$-ras ${ }^{G 12 D} ;$ Mox2-Cre mutants were produced at near Mendelian frequency at E12.5 ( $n=32 / 160 ; 20 \%)$ and at lower frequency at E13.5 $(n=16 / 105 ; 15 \%)$, developmental stages at which $\mathrm{K}$-ras ${ }^{G 12 D}$;Prm-Cre embryos are never recovered (Tuveson et al. 2004). Importantly, the yolk sacs and placentas of LSL-K-ras ${ }^{G 12 D}$;Mox2-Cre mutants were indistinguishable from wild-type controls (Fig. 2B,C). Similar results were obtained through a second, independent approach involving injection of K-ras ${ }^{G 12 D}$ ES cells into wild-type tetraploid blastocysts in order to restrict expression of K-ras ${ }^{G 12 D}$ to the embryonic compartment (data not shown). Taken together, 


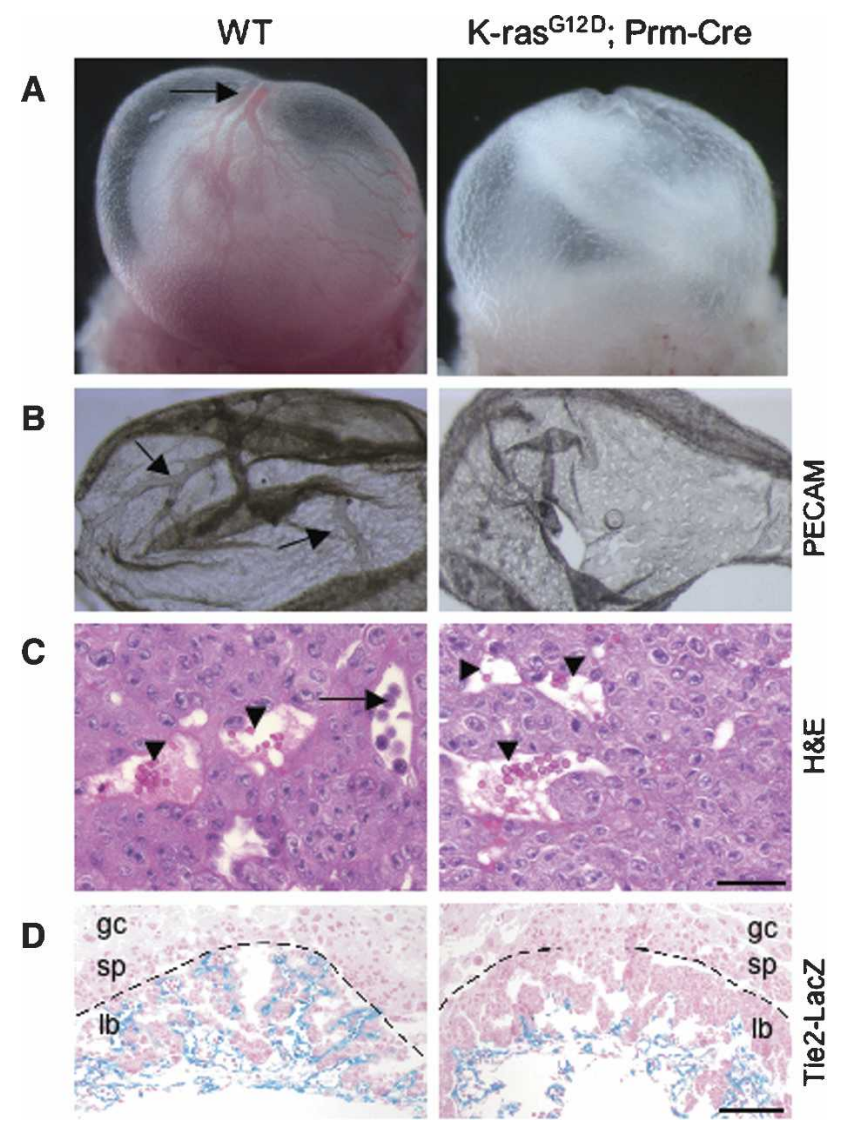

Figure 1. Extraembryonic phenotype of germline K-ras ${ }^{G 12 D}$ mutants. (A) Intact visceral yolk sacs of wild-type (WT) and LSL-K-ras ${ }^{G 12 D}$;Prm-Cre embryos at E9.5. Large vitelline vessels are readily apparent in wild-type yolk sacs (arrow), but are absent from mutant yolk sacs. (B) Whole-mount staining of E9.5 yolk sacs with anti-PECAM antibody, a specific marker of differentiated endothelial cells. Arrows highlight yolk sac vitelline vessels. (C) H\&E staining of E9.5 placentas. Fetal vessels containing nucleated embryonic erythroblasts (arrow) and maternal sinusoids containing adult enucleated red cells (arrowhead) are both present in the developing labyrinth of wild-type (WT) placentas. Mutant placentas contain maternal sinusoids (arrowhead), but lack fetal vessels. (D) LacZ staining of E9.5 placentas. Both wild-type (WT) and K-ras ${ }^{G 12 D}$ embryos carry a Tie2-LacZ transgene to mark endothelial cells. Sections were counterstained with nuclear fast red. The dashed line demarcates the labyrinth layer of the placenta. (lb) Labyrinth; (sp) spongiotrophoblast layer; (gc) giant cell layer. Bars: $C, 25 \mu \mathrm{m} ; D, 100 \mu \mathrm{m}$.

these findings indicate that the primary defect in germline $\mathrm{K}$-ras ${ }^{G 12 D}$ mutants is due to abnormalities in the placenta, leading to placental insufficiency and early embryonic lethality.

Germline mutants expressing oncogenic K-ras in the embryo proper die of cardiovascular and hematopoietic defects

Germline K-ras ${ }^{G 12 D}$ embryos supplied by functionally normal placentas bypass the lethality due to trophoblast dysfunction, but still die by E14.5. At E12.5, mutants appeared grossly normal in appearance (Fig. 2D), but rapidly developed pallor, edema, and peripheral hemorrhages (Fig. 2E), consistent with heart failure. To determine the cause of heart failure, we compared the anatomy of control and K-ras ${ }^{G 12 D}$ hearts at E13.5. All mutant hearts exhibited prominent ventricular septal defects, frequently in conjunction with double outlet right ventricle, excess endocardial cushion tissue, leading to outflow tract obstruction, and atrioventricular valve malformation (Supplementary Fig. 3A,B; data not shown). These defects are highly reminiscent of the cardiac phenotype of mouse mutants lacking the Ras-GAPrelated protein neurofibromin (Lakkis and Epstein 1998), and confirm a critical role for K-ras activation in cardiac organogenesis.

In addition to heart defects, $K$-ras ${ }^{G 12 D}$ embryos also exhibited fetal liver defects, with extensive areas of apoptotic cell death superimposed on a hypocellular background (Supplementary Fig. 3C,D). In colony formation assays, fetal-liver-derived hematopoietic progenitors expressing activated K-ras formed larger colonyforming unit-erythroid (CFU-E) and burst-forming uniterythroid (BFU-E) colonies compared with wild-type controls (data not shown). However, mutant red cells appeared very immature and occasionally highly atypical (Supplementary Fig. 3E,F), consistent with a block in erythroid differentiation. Similar results have been observed when fetal liver erythroblasts are infected with retroviruses encoding oncogenic H-ras (Zhang et al. 2003) and when $K$-ras ${ }^{G 12 D}$ is expressed in adult erythroid cells (Braun et al. 2006). Thus, K-ras ${ }^{G 12 D}$ embryos likely die of heart failure as well as anemia.

\section{Oncogenic K-ras activation causes defective fetal lung} branching morphogenesis

In addition to marked defects in cardiovascular and hematopoietic development, germline activation of K-ras induces a striking fetal lung phenotype. In contrast to wild-type E12.5 fetal lungs, with secondary and tertiary bronchi visible within the lung lobes, $K$-ras ${ }^{G 12 D}$ lungs contained large dilated bronchi (Fig. 3A), suggestive of a lung branching defect. This defect was more pronounced at E14.5, when, instead of the elaborate branching network of controls, mutant lungs showed persistent dilated bronchi with few terminal bronchi or bronchioles (data not shown). To confirm a specific defect in lung branching morphogenesis, we examined the branching patterns of wild-type and $K$-ras ${ }^{G 12 D}$ fetal lungs in vivo and in vitro. Mutant lungs exhibited defective branching as early as E11.5, with significantly fewer branches compared with controls (Fig. 3B). This decrease in branching was associated with the formation of large, fluid-filled sacs in place of normal terminal branches (Fig. 3C). Similar results were obtained when E11.5 fetal lungs were cultured in vitro (Fig. 3D; data not shown).

Lung branching morphogenesis is a complex developmental process involving reciprocal interactions between the lung epithelium and its underlying mesen- 
A
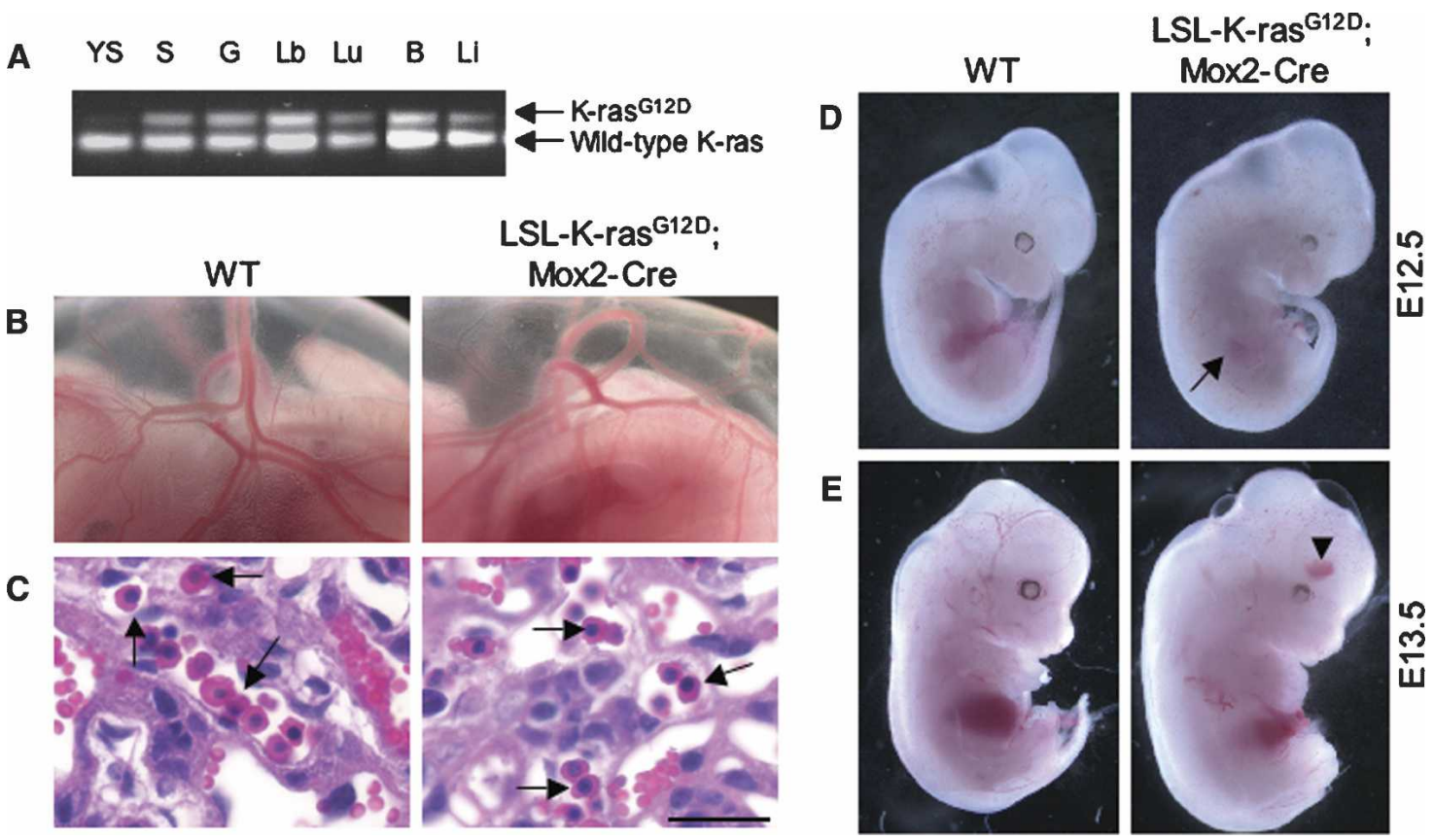

Figure 2. Rescue of early embryonic lethality by reconstitution of $K$-ras ${ }^{G 12 D}$ embryos with wild-type placentas. (A) PCR of genomic DNA from various tissues of $L S L-K-r a S^{G 12 D}$;Mox2-Cre embryos showing recombination of the LSL element. The recombined $K$-ras ${ }^{G 12 D}$ allele is readily distinguished from the wild-type (WT) K-ras allele by the presence of a single loxP site. Less recombination is detected in the yolk sac due to the extraembryonic endoderm present in this tissue. (Y) Yolk sac; $(\mathrm{S})$ skin; $(\mathrm{G})$ gut; (Lb) limb; $(\mathrm{Lu})$ lungs; (B) brain; (Li) liver. (B) Intact visceral yolk sacs of wild-type (WT) and LSL-K-ras ${ }^{\text {G12D }}$;Mox2-Cre embryos at E12.5. A welldeveloped vascular network is present in both wild-type and mutant yolk sacs. $(C)$ H\&E staining of E12.5 placentas. Placentas supporting $L S L$-K-ras ${ }^{G 12 D}$;Mox2-Cre embryos show normal vascularization of the labyrinth. Arrows indicate examples of nucleated embryonic erythroblasts. Bar, $25 \mu \mathrm{m}$. (D) Wild-type (WT) and K-ras ${ }^{G 12 D}$ embryos at E12.5 dissected free of the yolk sacs. Mutants appear morphologically normal, except for mild pallor of their fetal livers (arrow). (E) Wild-type (WT) and K-ras ${ }^{G 12 D}$ embryos at E13.5. Mutants now exhibit pallor, generalized edema, and peripheral hemorrhages (arrowhead).

chyme. To determine whether the branching defect identified in K-ras mutants resides in the epithelial or mesenchymal compartment of the lung, we used a doxycycline-inducible, Cre-regulated system to restrict expression of oncogenic K-ras to the developing lung epithelium (Perl et al. 2002). In this system, the reverse tetracycline transactivator (rtTA) is expressed in epithelial cells under the control of the human surfactant protein C (SP-C) promoter. In the presence of doxycycline, the rtTA activates expression of a tet-responsive Cre transgene [(tetO)7-Cre], leading to Cre-mediated recombination of a target gene throughout the developing lung epithelium.

As shown in Figure 3E, LSL-K-ras ${ }^{G 12 D}$;SPC-rt$T A$; (tet $O)_{7}$-Cre compound mutants exposed to doxycycline beginning at E6.5 demonstrated a dramatic lung branching defect similar to that observed in $L S L-K$ ras $^{\text {G12D }}$;Mox2-Cre mutants (Fig. 3A). This close resemblance in phenotype suggests that the defect in lung branching resides in the epithelial as opposed to the mesenchymal compartment. Because oncogenic activation of $\mathrm{K}$-ras is limited to the lung epithelium, LSL-Kras $^{G 12 D}$; SPC-rtTA; (tetO) $)_{7}$-Cre mutants bypass the lethality due to cardiac and hematopoietic deficiencies and can be recovered at later stages of gestation. At E16.5, K-ras mutant lungs exhibited an even more marked de- fect in branching, with large, epithelial-lined pouches in place of the numerous fine branches normally present at this stage (Fig. 3F). This defect in branching persisted through late gestation (Fig. 3G,H), leading to death in the early postnatal period (data not shown).

\section{Defective lung branching morphogenesis is accompanied by changes in epithelial cell differentiation but not proliferation or apoptosis}

To characterize the observed defect in lung branching at the cellular level, we first examined the proliferative rate of the developing lung epithelium. Surprisingly, wildtype and $\mathrm{K}$-ras ${ }^{G 12 D}$-expressing lung epithelial cells demonstrated similar levels of proliferation, based on immunohistochemical staining for the mitosis marker phospho-histone $\mathrm{H} 3$ (pHH3) (Fig. 4A,C) and BrdU incorporation, a marker of cells in S phase (Fig. 4B,C). Both wild-type and mutant fetal lungs also exhibited no evidence of apoptosis, based on cleaved caspase 3 (CC3) immunostaining (Fig. 4D). Thus, in the context of early lung development, oncogenic activation of K-ras does not appear to alter epithelial proliferation or cell survival.

To determine the effect of oncogenic K-ras on lung epithelial differentiation, we first examined expression 


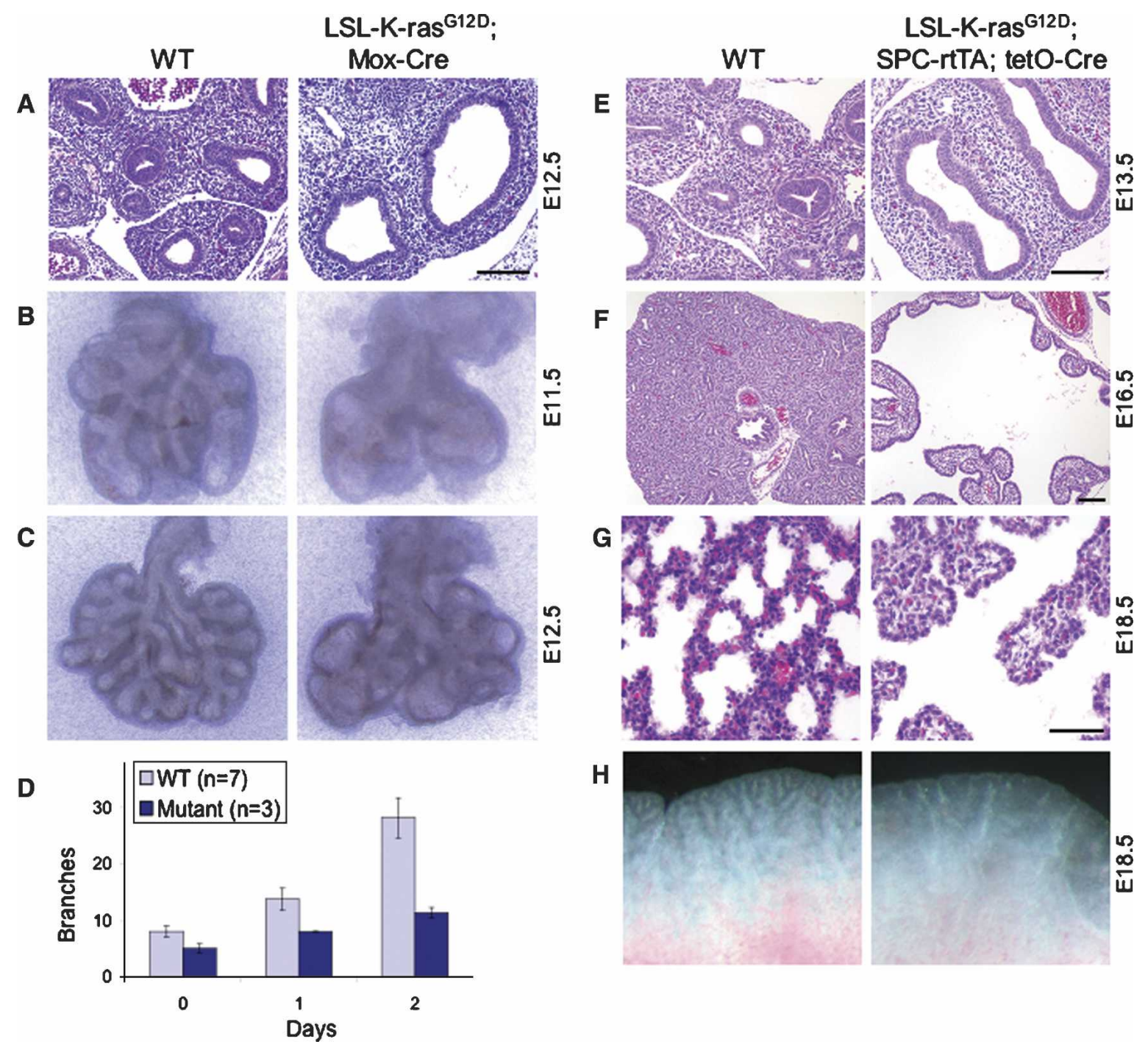

Figure 3. Disruption of lung epithelial branching morphogenesis by germline activation of $K$-ras. (A) H\&E-stained transverse sections of E12.5 fetal lungs from wild-type (WT) and $K$-ras ${ }^{G 12 D}$ embryos. $(B, C)$ Lung branching patterns at E11.5 (B) and E12.5 (C). Shown are freshly isolated fetal lungs from wild-type (WT) and $K$-ras ${ }^{G 12 D}$ embryos. $(D)$ Quantitation of terminal branches. Lungs were isolated from E11.5 embryos and cultured in vitro for $2 \mathrm{~d}$. Results are means \pm SEM. $(E-G)$ H\&E-stained sections of fetal lungs from wild-type (WT) and LSL-K-ras ${ }^{G 12 D}$; SPC-rtTA; (tetO) $)_{7}$-Cre embryos at E13.5 $(E)$, E16.5 $(F)$, and E18.5 $(G)$. At E13.5, doxycycline-treated triple mutants exhibit a severe defect in lung branching similar to that of germline $K$-ras ${ }^{G 12 D}$ mutants shown in $A$. $(H)$ Freshly isolated lungs from wild-type (WT) and LSL-K-ras ${ }^{G 12 D}$; SPC-rtTA; (tetO) ${ }_{7}$-Cre embryos at E18.5 showing persistence of the lung branching phenotype. Bars: $A, E, F, 100 \mu \mathrm{m} ; G, 50 \mu \mathrm{m}$.

of the epithelial cell marker thyroid transcription factor-1 (Titf1 or TTF-1) (Lazzaro et al. 1991). At E12.5, both wild-type and K-ras mutant fetal lungs demonstrated uniform expression of Titf1 in the primitive bronchial epithelium (data not shown). As markers of distinct lung epithelial cell lineages are not readily detectable until late gestation, we examined doxycycline-treated $L S L-K$ ras $^{G 12 D}$;SPC-rtTA; (tetO) ${ }_{7}$-Cre compound mutants at E18.5. Immunofluorescence staining of both wild-type and K-ras mutant fetal lungs showed strong expression of SP-C (Fig. 4E), suggestive of normal alveolar type II differentiation. However, consistent with early studies (Wert et al. 1993), SP-C expression was also detectable in undifferentiated progenitors, albeit at lower levels (data not shown), precluding definitive identification of type II cells in K-ras mutant lungs. Strikingly, expression levels of $\beta$-tubulin and CC10, markers of ciliated and Clara cell lineages, respectively, were significantly decreased in mutant compared with wild-type fetal lungs (Fig. 4F,G). In contrast, neuroendocrine differentiation, as assessed by immunohistochemical staining for calcitonin generelated peptide (CGRP), appeared normal in K-ras mutants, with no statistically significant change in numbers of CGRP-positive clusters per lobe (Fig. 4H). Based on cell labeling experiments demonstrating that the $S P C$-rtTA transgene is not expressed in neuroendocrine 

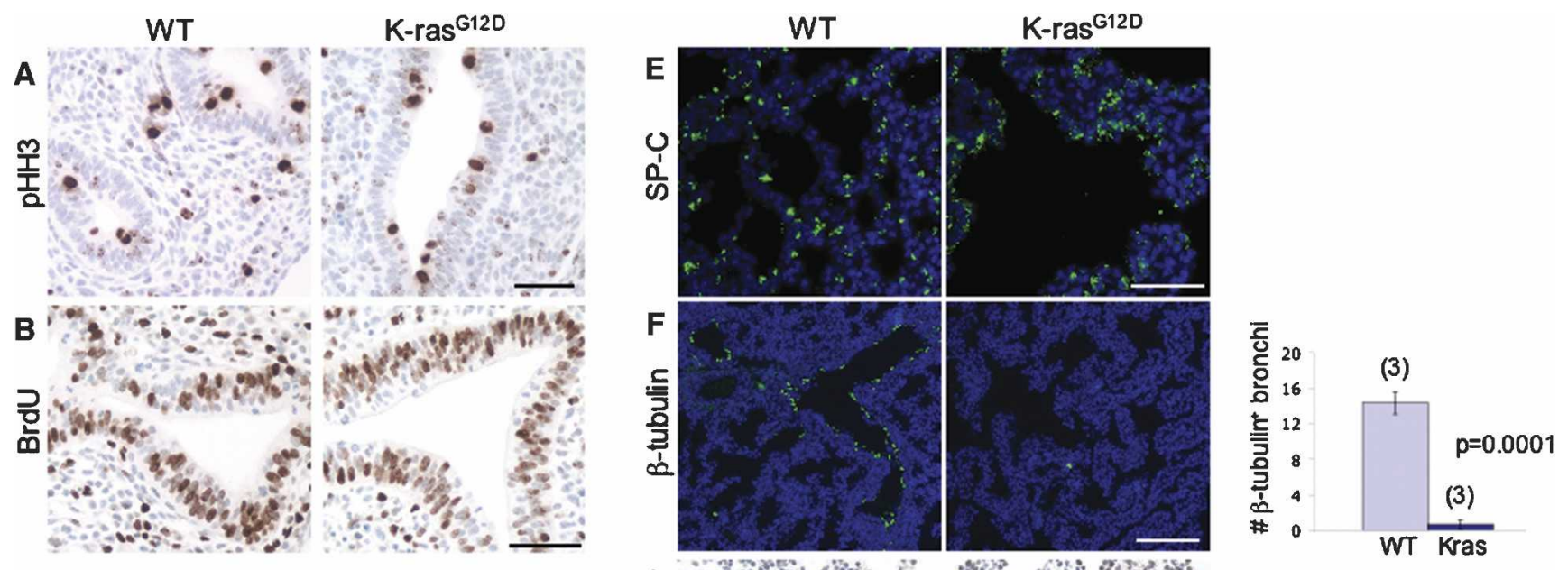
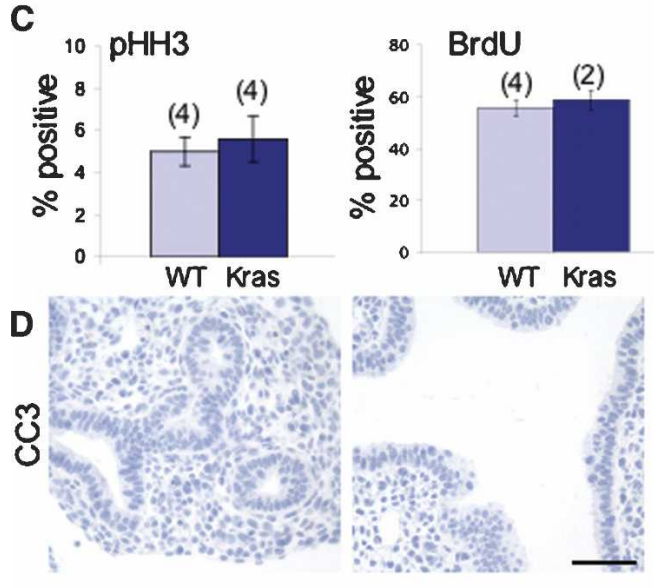
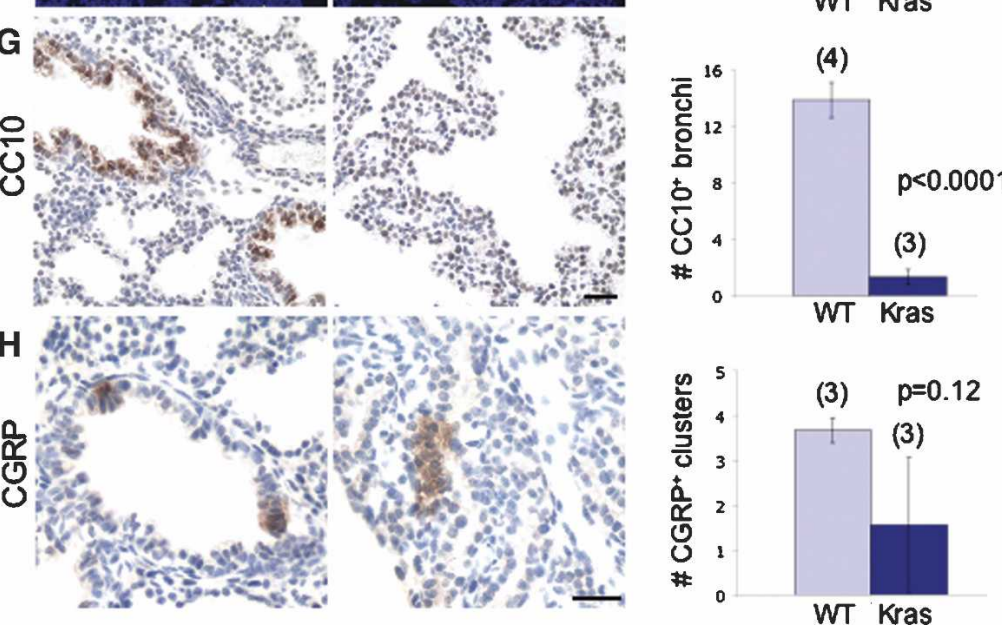

Figure 4. Proliferation, cell survival, and differentiation in $K$-ras ${ }^{G 12 D}$ fetal lungs. (A) Immunostaining of E12.5 fetal lungs for phosphohistone H3. (B) Immunostaining of E12.5 fetal lungs for BrdU. (C) Quantitation of phospho-histone H3-positive and BrdU-positive cells in the lung epithelium. The numbers of embryos analyzed are in parentheses. More than 1000 epithelial cells were counted per embryo. Results are means \pm SEM. $(D)$ Immunostaining of E12.5 fetal lungs for the apoptosis marker CC3. Neither wild-type (WT) nor $K$-ras ${ }^{G 12 D}$ fetal lungs exhibit CC3-positive cells. $(E, F)$ SP-C and $\beta$-tubulin immunofluorescence of E18.5 fetal lungs from wild-type (WT) and LSL-K-ras ${ }^{G 12 D}$; SPC-rtTA; (tetO) ${ }_{7}$-Cre embryos, both treated with doxycycline from E6.5 to E16.5. The numbers of embryos analyzed are in parentheses. $(G, H)$ Immunohistochemical staining of E18.5 fetal lungs for CC10 and CGRP, markers of Clara cells and neuroendocrine cells, respectively. The numbers of embryos analyzed are in parentheses. In $F-H$, three step sections (100 $\mu \mathrm{m})$ were examined per embryo. The bar graphs in $F$ and $G$ show the number of positive bronchi per lobe, with positive bronchi containing at least one positive cell. Bars: $A, B, D, E, 50 \mu \mathrm{m} ; F, 100 \mu \mathrm{m} ; G, H, 25 \mu \mathrm{m}$.

cells of the lung (Perl et al. 2002), this finding may simply reflect the absence of SPC-directed, Cre-mediated recombination in this particular lineage. Taken together, our data indicate that oncogenic K-ras activation leads to defective lung branching morphogenesis and, at later stages, to a specific block in proximal (and possibly distal) lung epithelial differentiation.

The lung branching defect induced by oncogenic K-ras is associated with up-regulation of Sprouty-2 and abnormal MAP kinase localization

The disruption in branching morphogenesis suggested a potential role for one or more branching regulators in mediating the lung phenotype of germline K-ras mutants. Among the key regulators of branching, Spry pro- teins were of particular interest given previous studies demonstrating MAPK attenuation in the setting of physiologic levels of oncogenic K-ras (Guerra et al. 2003; Tuveson et al. 2004), and the well-established role of Spry proteins as negative-feedback inhibitors of Ras/ MAPK signaling. Specifically, Spry proteins have been shown to be induced by receptor tyrosine kinase (RTK) signaling (Minowada et al. 1999), and to antagonize RTKdependent activation of the Ras/MAPK pathway at multiple levels (Mason et al. 2006). We therefore hypothesized that germline activation of K-ras might result in Spry overexpression, leading to aberrant MAPK regulation and disruption of normal lung development.

To begin to test this hypothesis, we first examined the expression patterns of Spry genes in the developing lung by in situ hybridization. Whereas wild-type lungs 
showed variable expression of Spry-2, with high levels restricted to the leading edge of branching tips, K-ras ${ }^{G 12 D}$ lungs exhibited significantly stronger and more uniform expression of Spry-2 throughout much of the mutant epithelium (Fig. 5A). The expression levels of other Spry genes were similar in wild-type and K-ras ${ }^{G 12 D}$ fetal lungs, with Spry-1 localized in the epithelium (Fig. 5A) and Spry-4 detected in the distal lung mesenchyme (data not shown). In addition, two other branching regulators-Bmp4 and Shh-also showed similar levels of expression based on whole mount in situ hybridization studies (Supplementary Fig. 4A,B), whereas Fgf10 appeared to be moderately down-regulated in mutants compared with controls (Supplementary Fig. 4C). To confirm the specific induction of Spry proteins by oncogenic K-ras and exclude the possibility that Spry induction is a secondary consequence of defective lung branching, we performed immunoblotting of whole cell lysates from LSL-K-ras G12D MEFs. Activation of $K$-ras ${ }^{G 12 D}$ expression by treatment with Cre resulted in a significant increase in Spry-2, Spry-4, and, to a lesser extent, Spry-1 (Fig. 5B). In contrast, levels of phosphorylated Erk (phosphoErk or phosphoMAPK) were similar to decreased $K$-ras ${ }^{G 12 D}$-expressing MEFs compared with controls, consistent with previous observations /Guerra et al. 2003; Tuveson et al. 2004).

The striking up-regulation of the Ras/MAPK antagonist Spry-2 in the setting of oncogenic K-ras suggested that aberrant MAPK activation might underlie the lung branching defect of germline K-ras mutants. In support of this notion, several studies have demonstrated an important role for MAPK activation in proper branching morphogenesis of the lung and kidney (Fisher et al. 2001; Liu et al. 2004). We first determined levels of phospho-
A

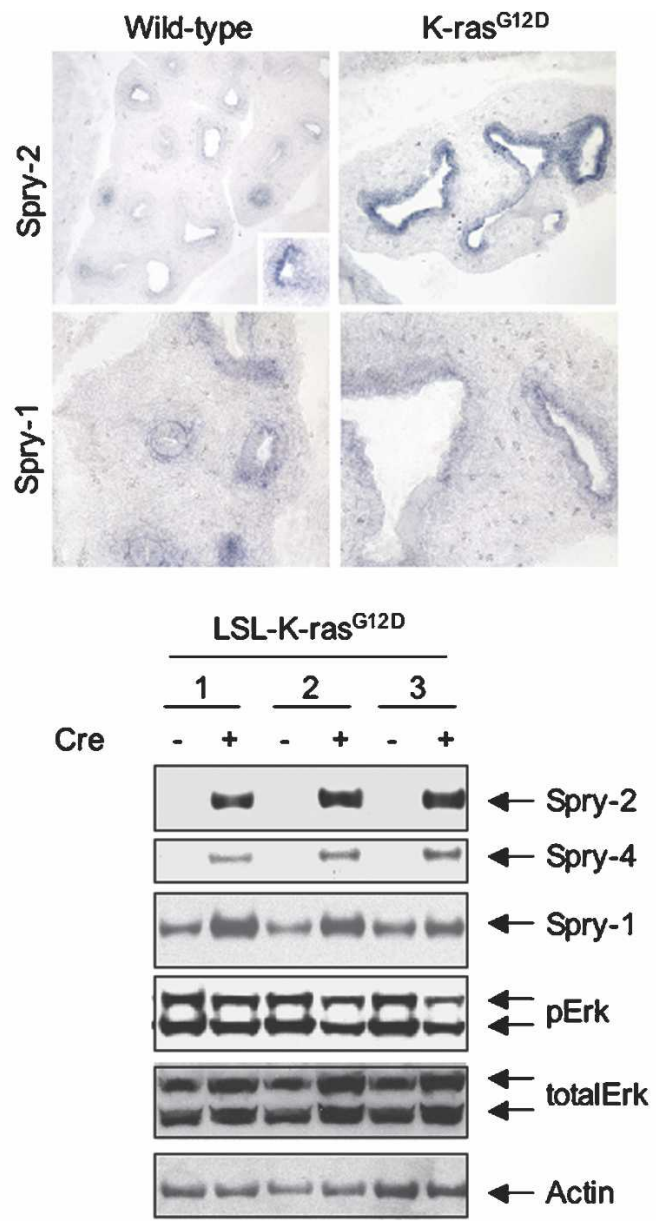

C

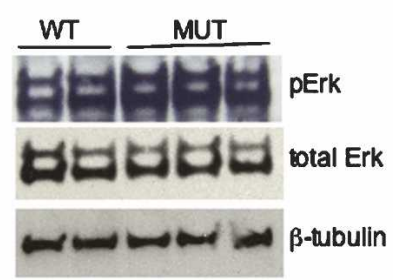

D

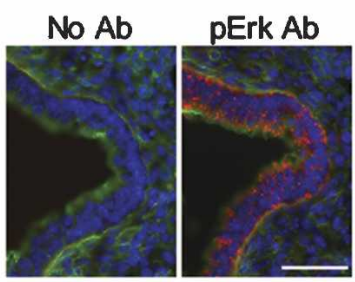

E
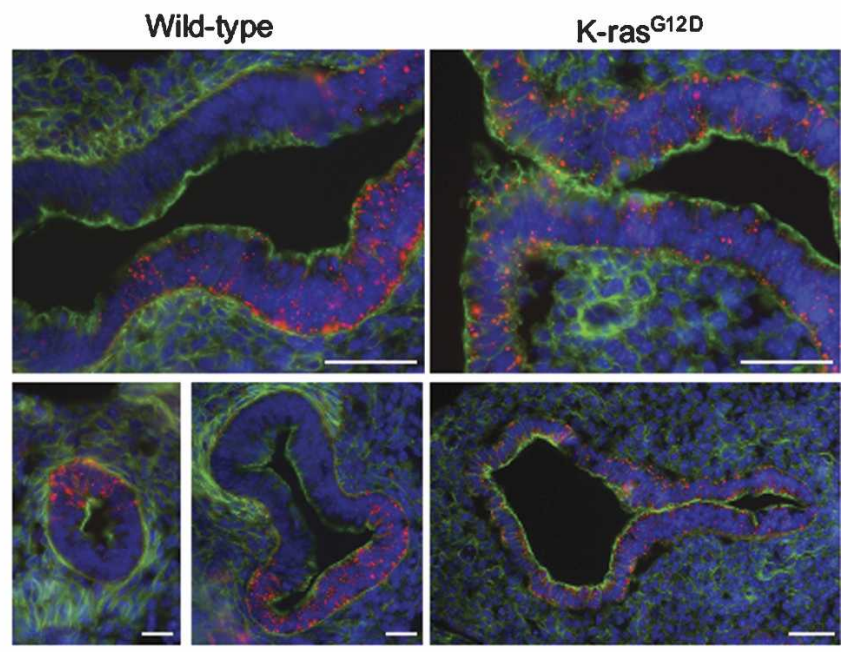

Figure 5. Induction of the Spry family of Ras/MAPK antagonists and aberrant MAPK activation in $K$-ras ${ }^{G 12 D}$ fetal lungs. $(A)$ In situ hybridization analysis of Spry expression in wild-type (WT) and K-ras ${ }^{G 12 D}$ fetal lungs at E13.5. (B) Spry protein expression in three independently derived, $L S L-K$-ras ${ }^{G 12 D}$ MEF lines, before and after treatment with Cre. $(C)$ Immunoblotting of whole-cell lysates of E12.5 fetal lungs for phosphoErk. (D) Immunoblotting of wild-type (WT) fetal lungs demonstrating the specificity of pErk staining (red). The serial sections (10 $\mathrm{m}$ apart) were photographed using the same exposure time. The actin cytoskeleton is stained in green with phalloidin; nuclei are stained blue with DAPI. (E) Immunohistochemical staining of E12.5 fetal lungs for phosphoErk. Wild-type (WT) lungs show a clear differential distribution of phosphoErk in the lung epithelium with sharp boundaries between areas of high and low Erk activity. In contrast, $K$-ras ${ }^{G 12 D}$ lungs show uniform pErk expression in the lung epithelium with no sharp boundaries identifiable. Bars: $D, 50 \mu \mathrm{m} ; E, 25 \mu \mathrm{m}$. 
and total MAPK by immunoblotting of whole cell lysates from wild-type and K-ras ${ }^{G 12 D}$ fetal lungs. Curiously, mutant fetal lungs contained equal to mildly increased levels of phosphoMAPK compared with wildtype fetal lungs (Fig. 5C). However, examination of expression patterns by immunohistochemistry revealed a profound difference in the distribution of phosphoMAPK. Whereas wild-type lungs demonstrated restricted areas of intense MAPK activation, particularly at the tips of initiating lung buds (Fig. 5E, left panels), mutants expressing oncogenic K-ras showed lower and relatively uniform expression of phosphoMAPK (Fig. 5E, right panels). This low-level, uniform distribution was observed throughout the entire mutant lung epithelium (Fig. 5E, right panels) and likely accounts for the elevated levels of phosphoMAPK detected by immunoblotting (Fig. 5C). These results suggest that the molecular mechanism by which oncogenic K-ras disrupts lung branching morphogenesis may involve Spry-mediated suppression of the differential distribution of MAPK activation required for normal lung bud initiation and outgrowth (Liu et al. 2004).

Lentiviral short hairpin RNA (shRNA)-mediated knockdown of Spry-2 in vivo rescues the lung branching defect induced by oncogenic K-ras

To rigorously test the role of Spry-2 induction in mediating the lung branching defect of K-ras ${ }^{G 12 D}$ embryos, we used conditional RNAi to abrogate Spry-2 up-regulation upon K-ras activation in vivo. We first derived mouse embryonic stem (ES) cells carrying the conditional LSL-K-Ras ${ }^{G 12 D}$ allele as well as the SPC-rtTA and (tet $\mathrm{O})_{7}$-Cre transgenes to allow doxycycline-inducible expression of Cre in the lung epithelium (Perl et al. 2002). Mutant ES cells were infected with lentivirus encoding GFP and a conditional, Cre-regulated, short hairpin RNA (shRNA) targeting Spry-2 (Ventura et al. 2004). Multiple, independent, GFP-positive ES clones were isolated and subsequently injected into wild-type tetraploid blastocysts. Recipient females were treated with doxycycline starting at E6.5, leading to activation of K-ras as well as knockdown of Spry-2 in the lung epithelium of developing embryos (Supplementary Fig. 5). For comparison, we also crossed the $L S L$-K-ras ${ }^{G 12 D}$ allele together with the Mox2-Cre transgene into a Spry-2-null background. Both the knockdown and knockout approaches yielded similar results and are summarized together.

On gross examination, knockdown mutant embryos were indistinguishable from control mutant embryos and showed uniform GFP expression (Fig. 6A; data not shown). However, compared with control infected K-ras ${ }^{G 12 D}$ lungs, K-ras ${ }^{G 12 D}$; Spry-2 $2^{K D}$ lungs exhibited increased branching, with significantly fewer dilated bronchi and more terminal branches (Fig. 6B,C; data not shown). Consistent with these findings, histologic analysis of serially sectioned E12.5 embryos revealed increased numbers of bronchi per section in knockdown mutants compared with control mutants (mean 5.8 vs 4.6) (Supplementary Fig. 6). Importantly, both control in- fected and knockdown lungs demonstrated elevated RasGTP levels in a Raf-GST pull-down assay (Fig. 6D). In addition, knockdown or knockout of Spry-2 in the absence of activated K-ras resulted in only mild fetal lung abnormalities (Supplementary Fig. 7; data not shown).

To establish the mechanistic role of Spry-2 at the molecular level, we examined expression levels and patterns of MAPK activation. Immunoblotting of whole cell lysates prepared from control mutant and knockdown mutant fetal lungs demonstrated efficient and specific knockdown of Spry-2 (but not Spry-1 or Spry-4), accompanied by augmented activation of MAPK signaling (Fig. $6 \mathrm{E}$; data not shown). Immunohistochemical staining confirmed increased levels of phosphoMAPK in the lung epithelium of knockdown mutants compared with control mutants (Fig. 6F). Remarkably, in knockdown but not control mutants, a subset of lung buds now showed differential distribution of MAPK activation, with highly localized MAPK activity adjacent to low levels of activity (Fig. 6F, right panel). Furthermore, by in situ hybridization, knockdown mutant lungs demonstrated increased Fgf10 levels, with more numerous sites of localized expression compared with control mutants (Fig. 6G). Thus, knockdown of Spry-2 in the setting of oncogenic K-ras significantly suppresses the lung branching defect as well as the underlying molecular abnormalities, indicating a critical role for Spry-2 induction in the K-ras ${ }^{G 12 D}$ phenotype.

Spry-2 functions as a tumor suppressor in K-ras ${ }^{G 12 D}$-mediated lung tumorigenesis

The functional interaction between oncogenic K-ras and Spry-2 during lung development suggested a potential role for Spry-2 in modulating the effects of oncogenic $\mathrm{K}$-ras in lung tumorigenesis. To address this possibility, we first examined expression levels of Spry family members in mouse lung tumors induced by oncogenic K-ras. Individual lung tumors were isolated from 3- to 4-moold mice harboring a "latent" (LA2) K-ras ${ }^{G 12 D}$ allele (Johnson et al. 2001). This allele is sporadically activated following a spontaneous recombination event, leading to expression of oncogenic K-ras and lung tumor formation in $100 \%$ of animals. Immunoblotting of whole cell lysates prepared from LA2 tumors demonstrated marked up-regulation of Spry-2 and, to a much lesser extent, Spry-1 compared with normal lung (Fig. 7A). In six out of seven tumors examined, the degree of Spry-2 up-regulation appeared to loosely correlate with histological grade of the tumor (Fig. 7B). More importantly, Spry-2 levels were inversely correlated with levels of phosphoMAPK, consistent with Spry-mediated inhibition of MAPK signaling.

To elucidate the role of Spry-2 induction in K-ras ${ }^{G 12 D}$. mediated lung tumorigenesis, we examined lung tumor development in the presence and absence of Spry-2. The $L S L-K-$ ras $^{G 12 D}$ strain was crossed to a strain carrying a conditional null allele of Spry-2 (referred to as Spry- $2^{F 1}$ ) (Shim et al. 2005) to generate compound mutants with the genotypes LSL-K-ras ${ }^{G 12 D}$; Spry- $2^{F 1 /+}$ and LSL-K- 
A

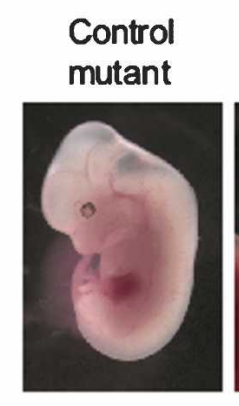

B

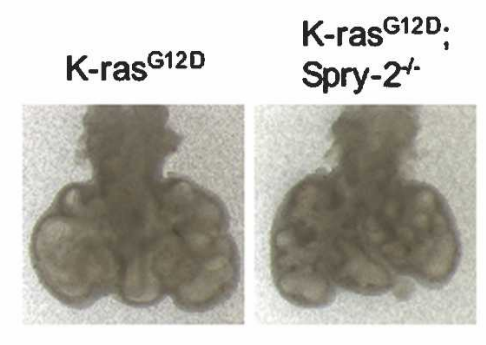

C

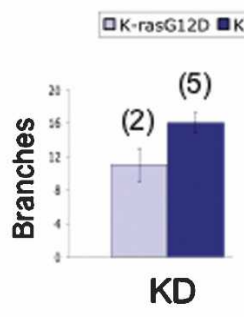

Knockdown mutant

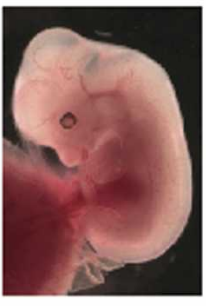

(1)

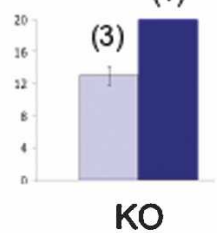

D

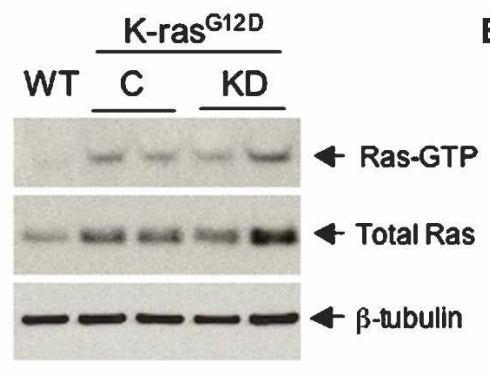

$\underline{\mathrm{K}-\mathrm{ras}^{\mathrm{G12D}}}$

E
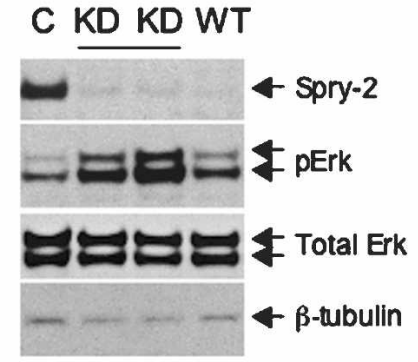

F
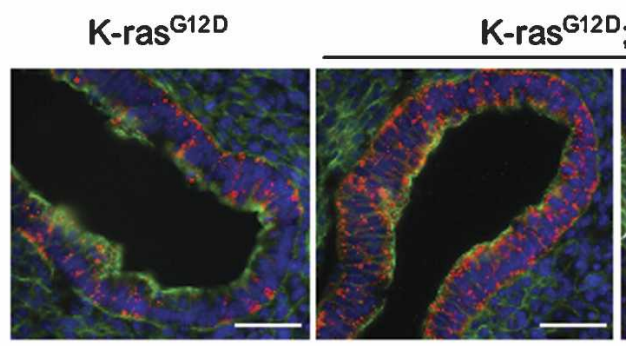

i Spry- $2^{\mathrm{KD}}$

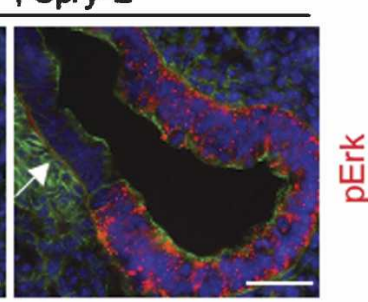

G
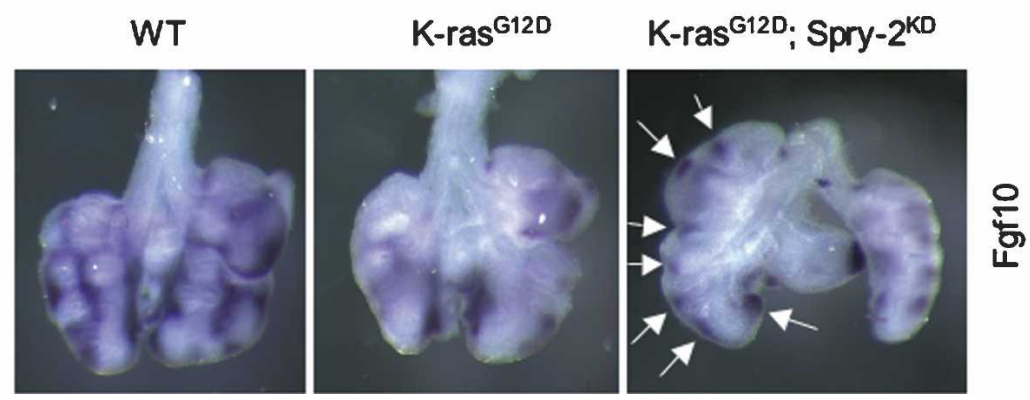

Figure 6. Suppression of the lung branching defect of $K$-ras ${ }^{G 12 D}$ embryos by lentiviral shRNA-mediated knockdown of Spry-2. (A) Gross appearance of control and knockdown mutant embryos. (B) Freshly isolated E12.5 lungs from LSL-K-ras ${ }^{G 12 D}$;Mox-Cre versus LSL-K-ras ${ }^{G 12 D}$;Mox-Cre; Spry-2-/- compound mutants. Lungs are photographed on a polyester membrane. (C) Quantitation of the rescue of lung branching by knockdown or knockout of Spry-2. Note that while Spry-2 knockdown or knockout increases the number of branches, the rescue is incomplete, as wild-type controls at E12.5 exhibit, on average, $>20$ terminal branches (see Fig. 3C). (D) Ras-GTP levels in fetal lungs derived from wild-type ES cells (WT), control infected LSL-K-ras ${ }^{G 12 D}$; SPC-rtTA; (tetO) $)_{7}$-Cre ES cells $(\mathrm{C})$, and $L S L-K$-ras ${ }^{G 12 D}$;SPC-rtTA; $(\text { tetO })_{7}$-Cre ES cells expressing the Spry-2 shRNA (KD). All embryos were treated with doxycycline starting at E6.5. (E) Immunoblotting of fetal lungs for Spry-2 expression confirming efficient knockdown of Spry-2 in the fetal lungs of LSL-K-ras ${ }^{G 12 D}$;SPC-rtTA; (tetO) $)_{-}$Cre; pSicoSpry-2 mutants. (F) Immunohistochemical staining of E12.5 fetal lung sections for phosphoErk. While $K$-ras ${ }^{G 12 D}$ control lungs always show low-level, uniform MAPK activation throughout the epithelium, $K$-ras ${ }^{G 12 D}$; Spry$2^{K D}$ lungs exhibit differential distribution of MAPK activity, with areas of intense activity juxtaposed with areas of low activity (arrow). Bar, $25 \mu \mathrm{m}$. (G) Whole-mount in situ hybridization of E12.5 fetal lungs. Compared with control K-ras mutants, K-ras mutants with the Spry-2 knockdown show increased Fgf10 expression, with numerous discrete areas of intense Fgf10 staining (arrows).

ras $^{G 12 D}$; Spry-2 $2^{F 1 / F 1}$. Lung tumors were induced by intranasal instillation of adenovirus expressing Cre (Jackson et al. 2001), and animals were sacrificed after 6 mo. Based on histologic analysis, both the number of tumors as well as the total tumor area (as a proportion of total lung area) were significantly increased in the absence of Spry-2 ( $p=0.0066$ and $p=0.025$, respectively) (Fig. 7C). These Spry-2-null tumors showed a similar broad spectrum of histologic grade as tumors expressing Spry-2 (Fig. 7D; data not shown). Immunohistochemical analysis demonstrated a mild increase in the number and intensity of phosphoErk-positive tumors (Fig. 7E,F). These re- sults support the notion that Spry-2 normally functions to limit tumor development and that the mechanism may involve antagonism of MAPK signaling.

\section{Discussion}

Despite several decades of intense study, the molecular basis of Ras oncogenicity remains incompletely understood. This lack of understanding likely stems from the complexity of the Ras signaling network. In addition to the canonical Ras effectors-Raf, PI3K, and RalGDS-at least seven other distinct Ras effector pathways have 
A

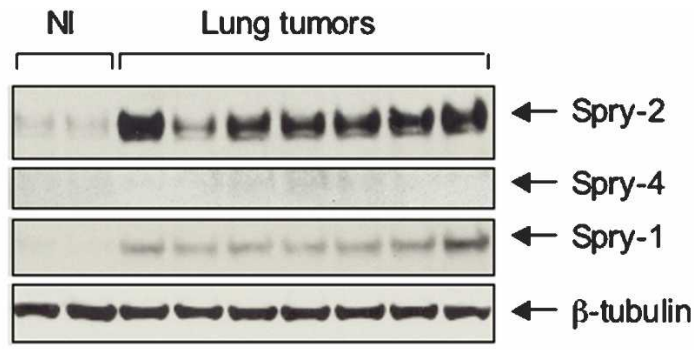

B

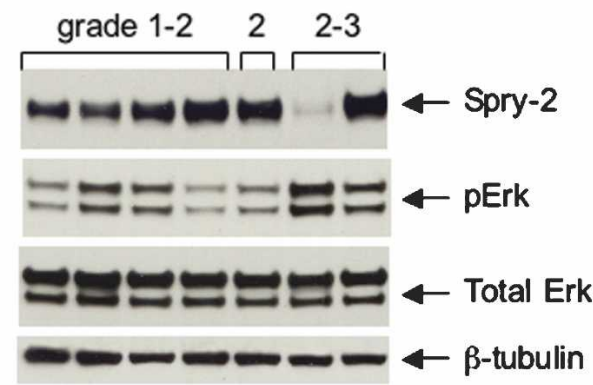

C

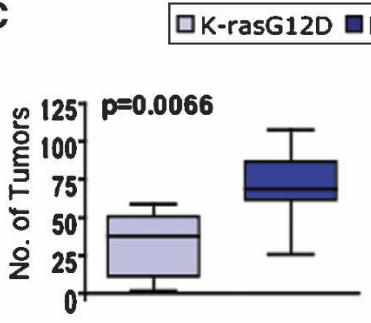

D

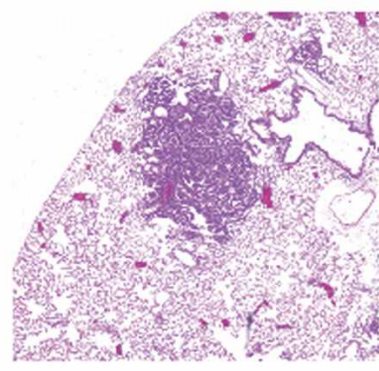

$\mathbf{E}$
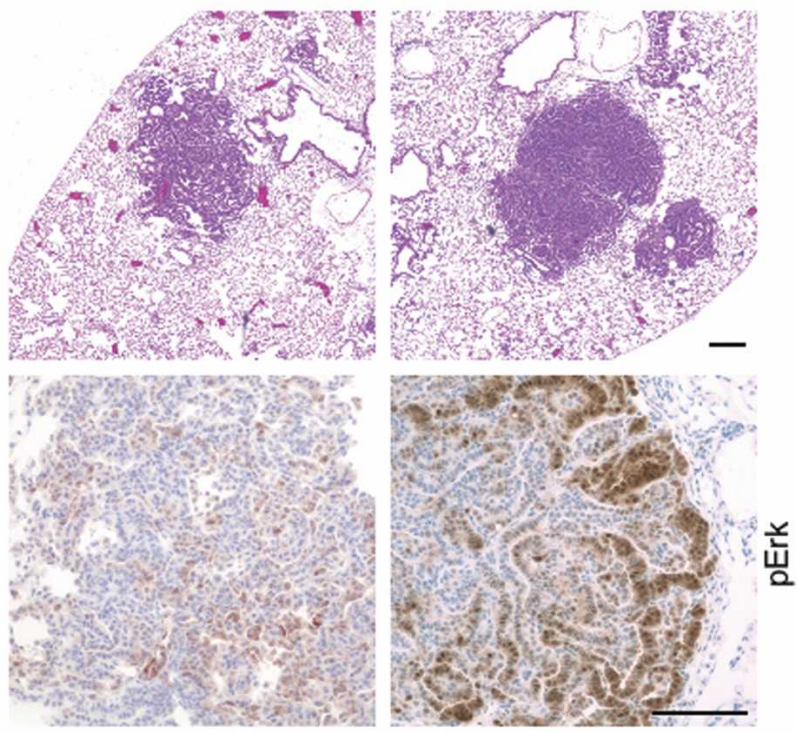

$\mathbf{F}$

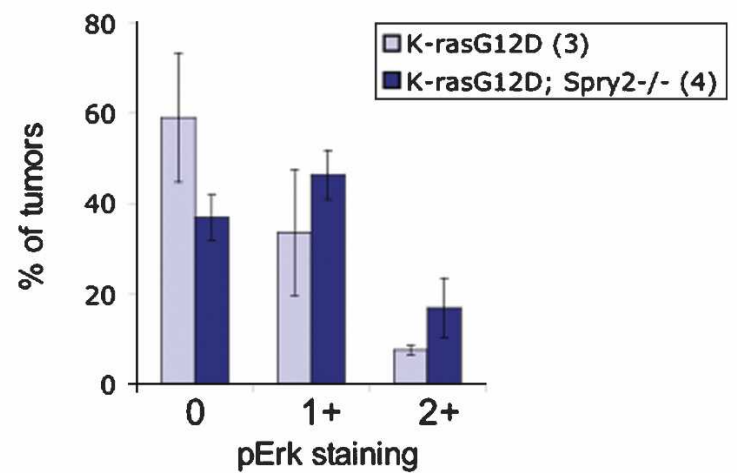

Figure 7. Tumor suppression by Spry-2 in $\mathrm{K}_{\text {-ras }}{ }^{G 12 D}$-mediated lung tumorigenesis. (A) Induction of Spry proteins in mouse lung tumors induced by oncogenic activation of K-ras. Individual tumors were dissected from LA2 mice (see text) and compared with normal lung tissue (NI) from wild-type littermates. (B) Expression of Spry-2 and phosphoMAPK in mouse lung tumors of different histological grades. $(C)$ Comparison of lung tumor number (left) and overall tumor burden (right) in $L S L-K-r a s{ }^{G 12 D}$ controls $(n=9)$ versus LSL-K-ras ${ }^{G 12 D}$; Spry- $2^{F 1 / F 1}$ mice $(n=17)$. Tumor burden is measured as a ratio of total tumor area to total lung area. Boxes represent interquartile ranges (between the 25th and 75th quartiles). The total range and median are also shown. (D) H\&E staining of lung tumors from $L S L$-K-ras ${ }^{G 12 D}$ and $L S L-K$-ras ${ }^{G 12 D}$; Spry- $2^{F l / F I}$ mice. (E) Immunostaining of lung tumors for phosphoErk. A significant subset of both K-ras control and K-ras;Spry-2-null tumors express phosphoErk. In the absence of Spry-2, there appear to be more tumors with intense pErk staining. $(F)$ Quantitation of pErk staining in K-ras control and K-ras;Spry-2-null lung tumors. pErk staining was scored based on the percentage of the tumor showing pErk expression: $0(<10 \%), 1+(10 \%-50 \%)$, and $2+(>50 \%)$. Results are means \pm SEM.

been defined (Malumbres and Barbacid 2003). Furthermore, none of the Ras effector pathways are simply linear cascades, and many are subject to feedback inhibition. The precise role of feedback inhibition and, importantly, the functional consequences of losing feedback inhibition have not been well studied. Here we demonstrate a critical role for one feedback inhibitor-Spry-2in modulating both the developmental and tumor phenotype associated with an oncogenic K-ras mutation. These results provide a paradigm for the regulation of oncogenic Ras signaling by Spry proteins and other feedback inhibitors.

\section{Negative feedback signaling in development}

In this study, we show that endogenous expression of oncogenic K-ras induces a profound defect in lung branching morphogenesis. This defect appears to be largely mediated by up-regulation of Spry-2, based on our ability to significantly suppress the defect by Spry-2 knockdown or knockout. The underlying mechanism most likely involves aberrant MAPK suppression and/or localization within the developing lung. MAPK activation has been shown to be required for branching morphogenesis of tubular epithelium, such as lung and kid- 
ney (Fisher et al. 2001; Liu et al. 2004). Moreover, based on studies in a three-dimensional culture system, the distribution of MAPK activation appears to be important for driving lung bud morphogenesis, with highest MAPK activity localized to the distal tips of endodermal buds (Liu et al. 2004). Consistent with this notion, fetal lungs expressing oncogenic K-ras demonstrate uniform expression of phosphoMAPK and markedly defective branching (Fig. 5E), whereas mutant lungs rescued by Spry2 knockdown now exhibit areas of differential MAPK activation (Fig. 6F). Recently, distinct roles for the two major MAPK isoforms, Erk1 (p44) and Erk2 (p42), have been reported (Vantaggiato et al. 2006). However, based on immunoblotting of fetal lungs, both MAPK isoforms appear to be similarly dysregulated by oncogenic K-ras in this particular developmental context (Fig. 5C).

The molecular mechanisms by which Spry proteins antagonize Ras/MAPK signaling are complex and highly dependent on cellular context. Based on numerous gainand loss-of-function studies, Spry proteins are capable of intercepting the Ras/MAPK pathway at multiple levels, from RTK to Raf activation. Additionally, although well established as negative modulators of RTK signaling, in certain mammalian contexts, Spry proteins fail to inhibit and may even potentiate EGFR signaling (Kim and Bar-Sagi 2004). In the setting of endogenous oncogenic K-ras, Spry-2 can clearly inhibit MAPK activation, as knockdown of Spry-2 in K-ras ${ }^{G 12 D}$ fetal lungs results in increased phosphoMAPK (Fig. 6E,F). One potential mode of Spry-2 action involves inhibition of MAPK signaling downstream from activated K-ras; for example, at the level of Raf activation. Both Spry-2 and Spry-4 have previously been shown to target Raf activation in 293T cell systems (Yusoff et al. 2002; Sasaki et al. 2003). A second potential mechanism involves interactions of Spry-2 with signaling components upstream of activated K-ras, leading to attenuation of FGFR but not EGFR signaling. In support of this hypothesis, phospho- and total EGFR levels are unchanged in wild-type versus $K$-ras ${ }^{G 12 D} \mathrm{mu}$ tant lungs (data not shown). Through suppression of FGF-dependent cell migration but maintenance of EGFregulated proliferation (Cabernard and Affolter 2005), the selective modulation of RTK-dependent MAPK activation could underlie the branching phenotype observed in $\mathrm{K}$-ras mutants.

Several findings suggest that other feedback inhibitors in addition to Spry-2 likely play an important role in regulating oncogenic K-ras signaling in development. First, oncogenic activation of K-ras in embryonic fibroblasts induces up-regulation of Spry-1, Spry-2, and Spry-4 (Fig. 5B). Gene expression profiling studies have confirmed up-regulation of Spry genes as well as revealed induction of Spreds (Spry-related proteins), MKPs (MAPK phosphatases), and other Ras regulators (A. Shaw, unpubl.). Similar results have recently been reported in the context of NF1 loss and Raf activation (Courtois-Cox et al. 2006), suggesting a global induction of negative feedback pathways by activated Ras. Second, while knockdown of Spry-2 significantly rescues the $K$-ras ${ }^{G 12 D}$-induced lung branching defect and restores lo- cal peaks of MAPK activity, the rescue is incomplete, with fewer terminal branches than wild-type controls and persistent mild to moderate dilatation. This inability to fully rescue the branching phenotype may be due to functional compensation by other Spry family members in the setting of Spry-2 knockdown. This hypothesis is supported by double knockout studies showing both nonredundant as well as redundant functions for the Spry proteins (Taniguchi et al. 2007). Of note, knockdown or knockout of Spry-2 did not rescue the defect in lung epithelial differentiation nor the cardiovascular or hematopoietic defects of K-ras ${ }^{G 12 D}$ embryos (data not shown). This may reflect different underlying molecular mechanisms and/or differential expression and utilization of feedback inhibitors in these different cellular contexts.

\section{Modeling human developmental disorders in the mouse}

In mammalian development, germline Ras mutations result in complex genetic syndromes. Mice harboring a germline mutation in K-ras display several features that overlap with the cognate human disorders, including congenital heart defects and blood disorders. However, mutant mice also exhibit unique defects in placental and lung development, neither of which has yet been reported in patients. This discordance in phenotype likely reflects the strength of the gain-of-function K-ras allele, with oncogenic G12 mutations conferring higher-level constitutive activation than the V14 or T58 substitutions identified in Noonan syndrome (Schubbert et al. 2006). While strong gain-of-function $H$-ras mutations are present in Costello syndrome (Aoki et al. 2005), the unique features of the K-ras ${ }^{G 12 D}$ phenotype may be due to differences in isoform expression and/or subcellular localization. Of note, few to no studies have actually examined the placentas or lungs of individuals with germline Ras mutations, raising the possibility of uncharacterized, subclinical defects. Whether negative feedback pathways are activated in these diseases and play a role in their pathogenesis remains to be determined.

\section{Tumor suppression by feedback inhibition}

Our data indicate that Spry-2 functions as a tumor suppressor in K-ras-driven lung cancer. This finding is consistent with several recent studies reporting down-regulation of Spry genes in breast, prostate, and liver cancer. In the case of prostate cancer, the degree of Spry-2 downregulation correlates with the invasiveness of the lesions, as well as with methylation status, suggesting epigenetic as opposed to mutational inactivation (McKie et al. 2005). In addition, forced expression of Spry proteins inhibits the growth and metastatic potential of a variety of cancer cell lines (for review, see Lo et al. 2006). Interestingly, in melanoma cell lines harboring the activating $B$-Raf V599E mutation, Spry-2 is up-regulated relative to wild-type B-Raf melanomas; however, Spry-2 acts as a 
tumor suppressor only in the wild-type melanomas (Tsavachidou et al. 2004). These findings suggest that the tumor-suppressive function of Spry proteins is very dependent on tumor context.

The mechanism of lung tumor suppression by Spry-2 is likely related to MAPK antagonism and inhibition of tumor proliferation. However, recent data also point to an alternative mechanism of tumor suppression, namely, induction of oncogene-induced senescence (Serrano et al. 1997; Lin et al. 1998). This cellular response has been most extensively studied in cultured cells, but has recently been confirmed in precancerous lesions from both mice and humans. While early studies suggested that senescence is mediated by sustained hyperactivation of Ras/MAPK signaling, one recent study proposes that the global suppression of Ras signaling pathways, in part through induction of Spry proteins, triggers the senescent response (Courtois-Cox et al. 2006). Of note, this study used NF1 knockdown and B-raf mutant cells, but not cells expressing endogenous, oncogenic Kras. We have shown that a similar negative feedback program is also induced in MEFs expressing endogenous K-ras ${ }^{G 12 D}$, yet these cells clearly do not undergo senescence (Tuveson et al. 2004). Thus, the role of negative feedback pathways as a general mechanism underlying oncogene-induced senescence requires further study and validation. Future efforts will focus on determining whether Spry proteins function as lung tumor suppressors by simply restraining excessive cellular proliferation or by enabling the senescence program.

\section{Materials and methods}

\section{Mouse strains}

The LSL-K-ras ${ }^{G 12 D}$ strain was interbred to Prm-Cre (O'Gorman et al. 1997), Mox2-Cre (Tallquist and Soriano 2000), Tie2-Cre (Jackson Laboratories), and SP-C/rtTA; (tetO) 7 -Cre mice (Perl et al. 2002). Mox2-Cre and Tie2-Cre transgenics were crossed to R26-LSL-LacZ mice (Jackson Laboratories) to determine patterns of Cre expression. LSL-K-ras ${ }^{G 12 D}$;Prm-Cre double mutants were crossed to Tie2-LacZ transgenics (Jackson Laboratories) to highlight vascular endothelial cells. $L S L-K$-ras ${ }^{G 12 D}$ mice were also bred to Spry-2 $2^{F l / F 1}$ and Spry-2 $2^{+/-}$mice (Shim et al. 2005). Details of PCR genotyping reactions are available on request.

\section{Histology, immunofluorescence, and immunohistochemistry}

Embryos were fixed in $4 \%$ PFA at $4{ }^{\circ} \mathrm{C}$ and embedded in paraffin. Serial $(5-\mu \mathrm{m})$ sections were cut and H\&E-stained. Immunofluorescence staining of tissues was performed essentially as described (Jackson et al. 2001) using antibodies directed against SP-C (Santa Cruz Biotechnology) and $\beta$-tubulin (Sigma). For standard IHC analysis, paraffin-embedded tissue was deparaffinized and unmasked using microwave heating in $0.01 \mathrm{M} \mathrm{Na}$ citrate buffer. The antibodies used were BrdU (BD Biosciences), phospho-histone H3 (Upstate Biotechnology), phosphoErk1/2 (Cell Signaling), cleaved caspase-3 (Cell Signaling), CC10 (gift from A. Mukherjee, National Institutes of Health, Bethesda, $\mathrm{MD}$ ), and CGRP (Peninsula Laboratories). For BrdU analysis, pregnant females were injected IP with $30 \mu \mathrm{g} / \mathrm{g}$ body weight, and embryos were collected $1 \mathrm{~h}$ later. For phosphoErk staining of embryonic lungs, lungs were fixed in $4 \%$ PFA for $2-4 \mathrm{~h}$ at $4^{\circ} \mathrm{C}$, frozen in OCT, and serially sectioned in their entirety $(10 \mu \mathrm{m})$. Unmasking was performed with $0.02 \%$ trypsin for $5 \mathrm{~min}$ at room temperature. The M.O.M. Immunodetection Kit (Vector Laboratories) was used together with mouse monoclonal antiphosphoErk1/2 antibody (Sigma). To detect phosphoErk, biotinylated secondary antibody was used, followed by Alexa Fluor 555-conjugated streptavidin (Invitrogen).

\section{In situ hybridization}

In situ hybridization analyses of frozen sections were performed as previously described. Spry probes were prepared from plasmids containing the complete protein region and $3^{\prime}$ untranslated region (UTR) (Minowada et al. 1999). Whole-mount in situ hybridization of fetal lungs was performed as described (Wilkinson 1992). The probes used were Bmp4 (Jones et al. 1991), Shh (Echelard et al. 1993), and Fgf10 (Bellusci et al. 1997).

\section{Hematopoietic progenitor assays}

See the Supplemental Material.

\section{Lung branching morphogenesis in vitro}

Lungs were isolated from E11.5 and E12.5 embryos and transferred to polyester membranes ( $3 \mu \mathrm{m}$ pore size) in six-well tissue culture plates (Costar). Lungs were cultured at $37^{\circ} \mathrm{C}$ at the airliquid interface over serum-free BGJb media (Invitrogen) supplemented with pen/strep, gln, and $0.20 \mathrm{mg} / \mathrm{mL}$ ascorbic acid (Sigma). Branching was monitored daily for up to $72 \mathrm{~h}$.

\section{ES cells}

ES cell lines were derived from blastocysts produced by crossing LSL-K-ras ${ }^{G 12 D}$ mice with Prm-Cre transgenics, as well as with SPC-rtTA; (tetO) ${ }_{7}$-Cre double transgenics. Blastocysts were explanted onto irradiated feeders and grown in ES medium with 50 uM PD98059 (Cell Signaling). Multiple (two to four) independent lines were obtained of the following genotypes: wild-type, K-ras ${ }^{G 12 D}$; Prm-Cre, and LSL-K-ras ${ }^{G 12 D}$;SPC-rtTA; (tetO) 7 -Cre.

\section{Cloning, design, and sequence of shRNAs}

shRNAs were designed using the pSico-Oligomaker 1.5 (developed by A. Ventura), which is freely available at http://web.mit. edu/ccr/labs/jacks/protocols/pSico.html. Conditional knockdowns were generated by cloning shRNAs into pSico as described (Ventura et al. 2004). The sequence of the Spry- 2 shRNA is 5'-GCCGGGTTGTCGTTGTAAA-3' and corresponds to nucleotides 1150-1168 of mSpry-2. The specificity of Spry-2 knockdown was confirmed by immunoblotting of whole-cell lysates with anti-Spry-1, anti-Spry-2, and anti-Spry-4 antibodies.

\section{Lentiviral infection of ES cells}

Lentiviral vectors encoding shRNAs were cotransfected with packaging vectors into $293 \mathrm{~T}$ cells as described (Rubinson et al. 2003). Supernatant was collected after $\sim 36 \mathrm{~h}$ and incubated with ES cells in single-cell suspensions. After $6 \mathrm{~h}$ at $37^{\circ} \mathrm{C}$, infected ES cells were washed and replated onto irradiated feeders in fresh ES medium. After 3-4 d, single GFP-positive clones were picked, expanded, and genotyped to confirm lentiviral integration. 


\section{Tetraploid complementation}

See the Supplemental Material.

\section{Western blot analysis and Ras-GTP assay}

Whole-cell lysates were prepared and immunoblotted as described (Tuveson et al. 2004). The antibodies used were Spry-2 (Abcam), Spry-1 and Spry-4 (Zymed), phospho- and total Erk1/2 (Cell Signaling), $\beta$-tubulin (Sigma), and actin (Santa Cruz Biotechnology). Ras-GTP was precipitated with Raf-GST and assessed according to the directions of the manufacturer (Upstate Biotechnology).

\section{Lung tumor induction and analysis}

Compound mutants were generated on a mixed 129/sv/FVBn background. Mice were infected with Ad-Cre as described (Jackson et al. 2001) and sacrificed after 6 mo. Lungs were isolated, fixed, and processed as described (Jackson et al. 2005). Lung and tumor areas were determined using Bioquant Image Analysis software in manual measurement mode.

\section{Acknowledgments}

We thank J. Whitsett for the SPC-rtTA;tetOCre mice, G. Minowada for the Spry-2 $2^{-/-}$and Spry2 $2^{F 1 / F 1}$ mice, S. O'Gorman for the Prm-Cre mice, and P. Soriano for the Mox2-Cre mice. We are very grateful to B. Hogan, E. Rawlins, Y. Liu, and B. Yu for discussion and technical advice, and A. Ventura for help with designing and cloning shRNAs. This work was supported by grants from the Damon Runyon Cancer Research Foundation to A.T.S. (DRG-100-04), and from the NCI to A.T.S. (5K08CA111634-2) and T.J. (5-UO1-CA84306-06). A portion of this work was supported by Cancer Center Support (Core) grant P30-CA14051. T.J. is an Investigator of the Howard Hughes Medical Institute.

\section{References}

Aoki, Y., Niihori, T., Kawame, H., Kurosawa, K., Ohashi, H., Tanaka, Y., Filocamo, M., Kato, K., Suzuki, Y., Kure, S., et al. 2005. Germline mutations in HRAS proto-oncogene cause Costello syndrome. Nat. Genet. 37: 1038-1040.

Bellusci, S., Grindley, J., Emoto, H., Itoh, N., and Hogan, B.L. 1997. Fibroblast growth factor 10 (FGF10) and branching morphogenesis in the embryonic mouse lung. Development 124: 4867-4878.

Bos, J.L. 1989. ras oncogenes in human cancer: A review. Cancer Res. 49: 4682-4689.

Braun, B.S., Archard, J.A., Van Ziffle, J.A., Tuveson, D.A., Jacks, T.E., and Shannon, K. 2006. Somatic activation of a conditional KrasG12D allele causes ineffective erythropoiesis in vivo. Blood 108: 2041-2044.

Cabernard, C. and Affolter, M. 2005. Distinct roles for two receptor tyrosine kinases in epithelial branching morphogenesis in Drosophila. Dev. Cell 9: 831-842.

Courtois-Cox, S., Genther Williams, S.M., Reczek, E.E., Johnson, B.W., McGillicuddy, L.T., Johannessen, C.M., Hollstein, P.E., Maccollin, M., and Cichowski, K. 2006. A negative feedback signaling network underlies oncogene-induced senescence. Cancer Cell 10: 459-472.

Downward, J. 2002. Targeting Ras signaling pathways in cancer therapy. Nat. Rev. Cancer 3: 11-22.

Echelard, Y., Epstein, D.J., St-Jacques, B., Shen, L., Mohler, J.,
McMahon, J.A., and McMahon, A.P. 1993. Sonic hedgehog, a member of a family of putative signaling molecules, is implicated in the regulation of CNS polarity. Cell 75: 14171430.

Esteban, L.M., Vicario-Abejon, C., Fernandez-Salguero, P., Fernandez-Medarde, A., Swaminathan, N., Yienger, K., Lopez, E., Malumbres, M., McKay, R., Ward, J.M., et al. 2001. Targeted genomic disruption of $\mathrm{H}$-ras and $\mathrm{N}$-ras, individually or in combination, reveals the dispensability of both loci for mouse growth and development. Mol. Cell. Biol. 21: 14441452.

Faria, T.N., Ogren, L., Talamantes, F., Linzer, D.I., and Soares, M.J. 1991. Localization of placental lactogen-I in trophoblast giant cells of the mouse placenta. Biol. Reprod. 44: 327-331.

Fisher, C.E., Michael, L., Barnett, M.W., and Davies, J.A. 2001. Erk MAP kinase regulates branching morphogenesis in the developing mouse kidney. Development 128: 4329-4338.

Guerra, C., Mijimolle, N., Dhawahir, A., Dubus, P., Barradas, M., Serrano, M., Campuzano, V., and Barbacid, M. 2003. Tumor induction by an endogenous K-ras oncogene is highly dependent on cellular context. Cancer Cell 4: 111-120.

Jackson, E.L., Willis, N., Mercer, K., Bronson, R.T., Crowley, D., Montoya, R., Jacks, T., and Tuveson, D.A. 2001. Analysis of lung tumor initiation and progression using conditional expression of oncogenic K-ras. Genes \& Dev. 15: 3243-3248.

Jackson, E.L., Olive, K.P., Tuveson, D.A., Bronson, R., Crowley, D., Brown, M., and Jacks, T. 2005. The differential effects of mutant p53 alleles on advanced murine lung cancer. Cancer Res. 65: 10280-10288.

Johnson, L., Greenbaum, D., Cichowski, K., Mercer, K., Murphy, E., Schmitt, E., Bronson, R.T., Umanoff, H., Edelmann, W., Kucherlapati, R., et al. 1997. K-ras is an essential gene in the mouse with partial functional overlap with N-ras. Genes \& Dev. 11: 2468-2481.

Johnson, L., Mercer, K., Greenbaum, D., Bronson, R.T., Crowley, D., Tuveson, D.A., and Jacks, T. 2001. Somatic activation of the K-ras oncogene causes early onset lung cancer in mice. Nature 410: 1111-1116.

Jones, C.M., Lyons, K.M., and Hogan, B.L. 1991. Involvement of Bone Morphogenetic Protein-4 (BMP-4) and Vgr-1 in morphogenesis and neurogenesis in the mouse. Development 111: 531-542.

Kim, H.J. and Bar-Sagi, D. 2004. Modulation of signalling by Sprouty: A developing story. Nat. Rev. Mol. Cell Biol. 5: 441-450.

Lakkis, M.M. and Epstein, J.A. 1998. Neurofibromin modulation of ras activity is required for normal endocardial-mesenchymal transformation in the developing heart. Development 125: 4359-4367.

Lazzaro, D., Price, M., de Felice, M., and Di Lauro, R. 1991. The transcription factor TTF-1 is expressed at the onset of thyroid and lung morphogenesis and in restricted regions of the foetal brain. Development 113: 1093-1104.

Lescisin, K.R., Varmuza, S., and Rossant, J. 1988. Isolation and characterization of a novel trophoblast-specific cDNA in the mouse. Genes \& Dev. 2: 1639-1646.

Lin, A.W., Barradas, M., Stone, J.C., van Aelst, L., Serrano, M., and Lowe, S.W. 1998. Premature senescence involving p53 and $\mathrm{p} 16$ is activated in response to constitutive MEK/MAPK mitogenic signaling. Genes \& Dev. 12: 3008-3019.

Liu, Y., Stein, E., Oliver, T., Li, Y., Brunken, W.J., Koch, M., Tessier-Lavigne, M., and Hogan, B.L. 2004. Novel role for Netrins in regulating epithelial behavior during lung branching morphogenesis. Curr. Biol. 14: 897-905.

Lo, T.L., Fong, C.W., Yusoff, P., McKie, A.B., Chua, M.S., Leung, H.Y., and Guy, G.R. 2006. Sprouty and cancer: The first 
terms report. Cancer Lett. 242: 141-150.

Malumbres, M. and Barbacid, M. 2003. RAS oncogenes: The first 30 years. Nat. Rev. Cancer 3: 459-465.

Mason, J.M., Morrison, D.J., Basson, M.A., and Licht, J.D. 2006. Sprouty proteins: Multifaceted negative-feedback regulators of receptor tyrosine kinase signaling. Trends Cell Biol. 16: $45-54$.

Matsui, T., Kinoshita, T., Morikawa, Y., Tohya, K., Katsuki, M., Ito, Y., Kamiya, A., and Miyajima, A. 2002. K-Ras mediates cytokine-induced formation of E-cadherin-based adherens junctions during liver development. EMBO J. 21: 1021-1030.

McKie, A.B., Douglas, D.A., Olijslagers, S., Graham, J., Omar, M.M., Heer, R., Gnanapragasam, V.J., Robson, C.N., and Leung, H.Y. 2005. Epigenetic inactivation of the human sprouty2 (hSPRY2) homologue in prostate cancer. Oncogene 24: $2166-2174$.

Minowada, G., Jarvis, L.A., Chi, C.L., Neubuser, A., Sun, X., Hacohen, N., Krasnow, M.A., and Martin, G.R. 1999. Vertebrate Sprouty genes are induced by FGF signaling and can cause chondrodysplasia when overexpressed. Development 126: 4465-4475.

Niihori, T., Aoki, Y., Narumi, Y., Neri, G., Cave, H., Verloes, A., Okamoto, N., Hennekam, R.C., Gillessen-Kaesbach, G., Wieczorek, D., et al. 2006. Germline KRAS and BRAF mutations in cardio-facio-cutaneous syndrome. Nat. Genet. 38: 294-296.

O'Gorman, S., Dagenais, N.A., Qian, M., and Marchuk, Y. 1997. Protamine-Cre recombinase transgenes efficiently recombine target sequences in the male germ line of mice, but not in embryonic stem cells. Proc. Natl. Acad. Sci. 94: 1460214607.

Perl, A.K., Wert, S.E., Nagy, A., Lobe, C.G., and Whitsett, J.A. 2002. Early restriction of peripheral and proximal cell lineages during formation of the lung. Proc. Natl. Acad. Sci. 99: 10482-10487.

Rodriguez-Viciana, P., Tetsu, O., Tidyman, W.E., Estep, A.L., Conger, B.A., Cruz, M.S., McCormick, F., and Rauen, K.A. 2006. Germline mutations in genes within the MAPK pathway cause cardio-facio-cutaneous syndrome. Science 311: $1287-1290$.

Rubinson, D.A., Dillon, C.P., Kwiatkowski, A.V., Sievers, C., Yang, L., Kopinja, J., Rooney, D.L., Ihrig, M.M., McManus, M.T., Gertler, F.B., et al. 2003. A lentivirus-based system to functionally silence genes in primary mammalian cells, stem cells and transgenic mice by RNA interference. Nat. Genet. 33: 401-406.

Sasaki, A., Taketomi, T., Kato, R., Saeki, K., Nonami, A., Sasaki, M., Kuriyama, M., Saito, N., Shibuya, M., and Yoshimura, A. 2003. Mammalian Sprouty4 suppresses Rasindependent ERK activation by binding to Raf1. Nat. Cell Biol. 5: 427-432.

Schlaeger, T.M., Bartunkova, S., Lawitts, J.A., Teichmann, G., Risau, W., Deutsch, U., and Sato, T.N. 1997. Uniform vascular-endothelial-cell-specific gene expression in both embryonic and adult transgenic mice. Proc. Natl. Acad. Sci. 94: 3058-3063.

Schubbert, S., Zenker, M., Rowe, S.L., Boll, S., Klein, C., Bollag, G., van der Burgt, I., Musante, L., Kalscheuer, V., Wehner, L.E., et al. 2006. Germline KRAS mutations cause Noonan syndrome. Nat. Genet. 38: 331-336.

Serrano, M., Lin, A.W., McCurrach, M.E., Beach, D., and Lowe, S.W. 1997. Oncogenic ras provokes premature cell senescence associated with accumulation of p53 and p16INK4a. Cell 88: 593-602.

Shim, K., Minowada, G., Coling, D.E., and Martin, G.R. 2005. Sprouty2, a mouse deafness gene, regulates cell fate deci- sions in the auditory sensory epithelium by antagonizing FGF signaling. Dev. Cell 8: 553-564.

Tallquist, M.D. and Soriano, P. 2000. Epiblast-restricted Cre expression in MORE mice: A tool to distinguish embryonic vs. extra-embryonic gene function. Genesis 26: 113-115.

Taniguchi, K., Ayada, T., Ichiyama, K., Kohno, R., Yonemitsu, Y., Minami, Y., Kikuchi, A., Maehara, Y., and Yoshimura, A. 2007. Sprouty2 and Sprouty4 are essential for embryonic morphogenesis and regulation of FGF signaling. Biochem. Biophys. Res. Commun. 352: 896-902.

Tsavachidou, D., Coleman, M.L., Athanasiadis, G., Li, S., Licht, J.D., Olson, M.F., and Weber, B.L. 2004. SPRY2 is an inhibitor of the ras/extracellular signal-regulated kinase pathway in melanocytes and melanoma cells with wild-type BRAF but not with the V599E mutant. Cancer Res. 64: 5556-5559.

Tuveson, D.A., Shaw, A.T., Willis, N.A., Silver, D.P., Jackson, E.L., Chang, S., Mercer, K.L., Grochow, R., Hock, H., Crowley, D., et al. 2004. Endogenous oncogenic K-rasG12D stimulates proliferation and widespread neoplastic and developmental defects. Cancer Cell 5: 375-387.

Vantaggiato, C., Formentini, I., Bondanza, A., Bonini, C., Naldini, L., and Brambilla, R. 2006. ERK1 and ERK2 mitogen-activated protein kinases affect Ras-dependent cell signaling differentially. J. Biol. 5: 14.

Ventura, A., Meissner, A., Dillon, C.P., McManus, M., Sharp, P.A., Van Parijs, L., Jaenisch, R., and Jacks, T. 2004. Cre-loxregulated conditional RNA interference from transgenes. Proc. Nat1. Acad. Sci. 101: 10380-10385.

Wert, S.E., Glasser, S.W., Korfhagen, T.R., and Whitsett, J.A. 1993. Transcriptional elements from the human SP-C gene direct expression in the primordial respiratory epithelium of transgenic mice. Dev. Biol. 156: 426-443.

Wilkinson, D.G. 1992. Whole mount in situ hybridization of vertebrate embryos. In In situ hybridization (ed. D.G. Wilkinson), pp. 75-83. IRL Press Oxford University, New York.

Wu, L., de Bruin, A., Saavedra, H.I., Starovic, M., Trimboli, A., Yang, Y., Opavska, J., Wilson, P., Thompson, J.C., Ostrowski, M.C., et al. 2003. Extra-embryonic function of Rb is essential for embryonic development and viability. Nature 421: 942-947.

Yusoff, P., Lao, D.H., Ong, S.H., Wong, E.S., Lim, J., Lo, T.L., Leong, H.F., Fong, C.W., and Guy, G.R. 2002. Sprouty2 inhibits the Ras/MAP kinase pathway by inhibiting the activation of Raf. J. Biol. Chem. 277: 3195-3201.

Zhang, J., Socolovsky, M., Gross, A.W., and Lodish, H.F. 2003. Role of Ras signaling in erythroid differentiation of mouse fetal liver cells: Functional analysis by a flow cytometrybased novel culture system. Blood 102: 3938-3946. 


\section{Erratum}

Genes \& Development 21: 694-707 (2007)

\section{Sprouty-2 regulates oncogenic K-ras in lung development and tumorigenesis}

Alice T. Shaw, Alexander Meissner, James A. Dowdle, Denise Crowley, Margaret Magendantz, Chensi Ouyang, Tiziana Parisi, Jayaraj Rajagopal, Leah J. Blank, Roderick T. Bronson, James R. Stone, David A. Tuveson, Rudolf Jaenisch, and Tyler Jacks

The authors wish to report an inadvertent error in the genetic backgrounds of the mice shown in Figure 7, C-F, involving strain imbalance and propagation of a tetOCre transgene with the Spry- $2^{F 1}$ allele. They have repeated the experiment using new cohorts of compound mutants lacking tetOCre. The corrected figure is shown below. Of note, at $12 \mathrm{wk}$ after Ad-Cre infection, the total number of tumors as well as overall tumor burden were both increased in $L S L-K-r a S^{G 12 D}$; Spry- $2^{F / / F I}$ mice $(n=4)$ compared with $L S L-K-r a S^{G 12 D}$; Spry-2 $2^{F l /+}$ controls $(n=7)$, although the difference was not statistically significant (Fig. 7C; data not shown). However, there was a statistically significant increase in the size of lung tumors lacking Spry-2 $(n=255)$ compared with control tumors $(n=243)$ (Fig. 7C,D). In addition, there was also a trend toward increased pErk staining in the absence of Spry-2 (Fig. 7E,F), as shown previously. This correction does not change the major findings or conclusions of the study.

A

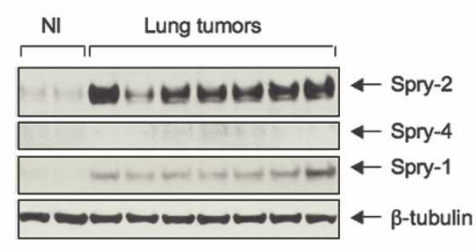

B

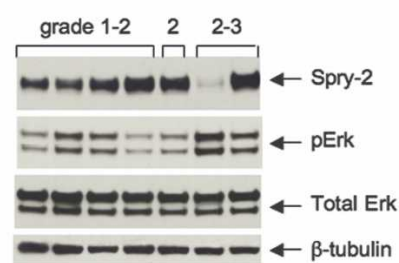

C

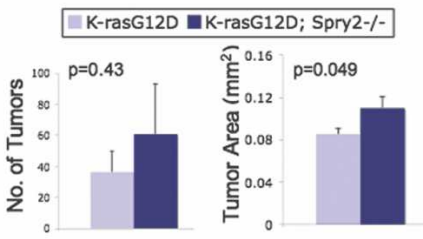

D
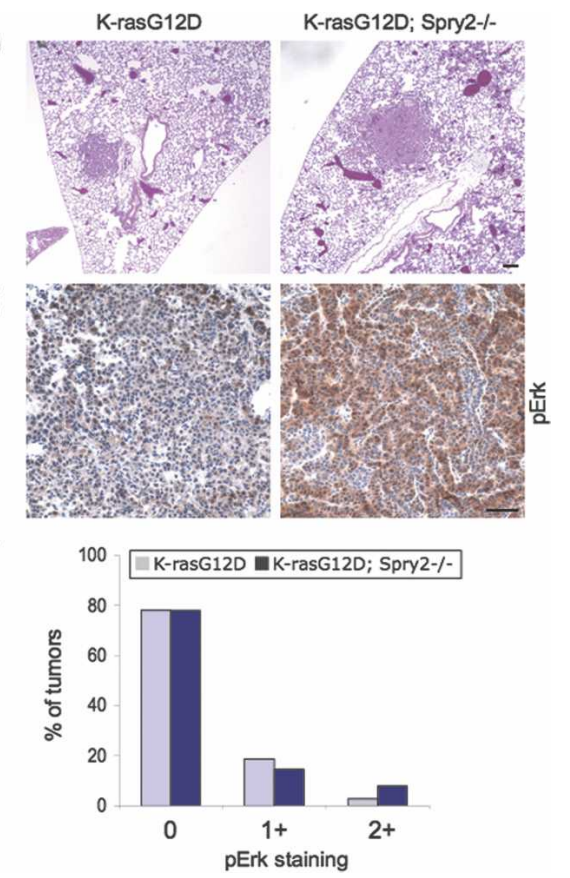


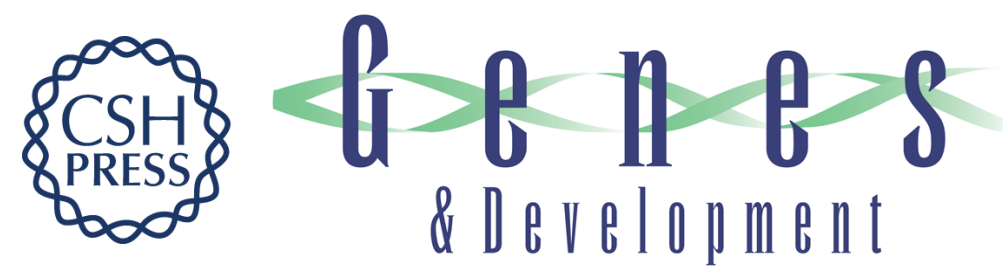

\section{Sprouty-2 regulates oncogenic K-ras in lung development and tumorigenesis}

Alice T. Shaw, Alexander Meissner, James A. Dowdle, et al.

Genes Dev. 2007, 21:

Access the most recent version at doi:10.1101/gad.1526207

\section{Supplemental http://genesdev.cshlp.org/content/suppl/2007/03/12/21.6.694.DC1 Material}

Related Content

References

\section{License}

Email Alerting

Service

\section{Erratum}

Genes Dev. January , 2008 22: 277

This article cites 48 articles, 23 of which can be accessed free at: http://genesdev.cshlp.org/content/21/6/694.full.html\#ref-list-1

Articles cited in:

http://genesdev.cshlp.org/content/21/6/694.full.htmI\#related-urls

Receive free email alerts when new articles cite this article - sign up in the box at the top right corner of the article or click here.

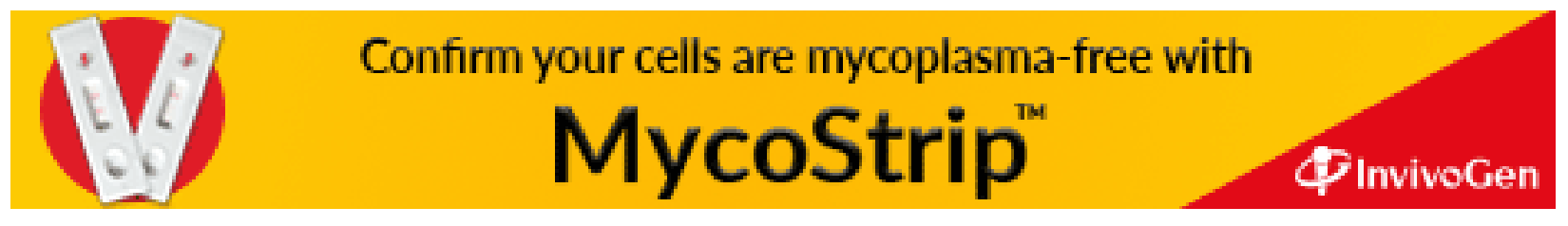

